\title{
Long non-coding RNA LINC01234 regulates proliferation, migration and invasion via HIF-2 $\alpha$ pathways in clear cell renal cell carcinoma cells
}

\author{
Feilong Yang ${ }^{1}$, Cheng Liu ${ }^{1}$, GuoJiang Zhao ${ }^{1}$, Liyuan Ge ${ }^{1}$, Yimeng Song ${ }^{1}$, Zhigang Chen ${ }^{1}$, Zhuo Liu ${ }^{1}$, Kai Hong \\ Corresp., 1 , Lulin Ma ${ }^{\text {Corresp. } 1}$ \\ ${ }^{1}$ Department of Urology, Peking University Third Hospital, Beijing, China \\ Corresponding Authors: Kai Hong, Lulin Ma \\ Email address: kenhong99@hotmail.com, malulin@medmail.com.cn
}

Long non-coding RNAs (IncRNAs) have been proved to have an important role in different malignancies including clear cell renal cell carcinoma (ccRCC). However, their role in disease progression is still not clear. The objective of the study was to identify IncRNAbased prognostic biomarkers and further to investigate the role of one IncRNA LINC01234 in progression of $\mathrm{CCRCC}$ cells. We found that six adverse prognostic IncRNA biomarkers including LINC01234 were identified in cCRCC patients by bioinformatics analysis using The Cancer Genome Atlas (TCGA) database. LINC01234 knockdown impaired cell proliferation, migration and invasion in vitro as compared to negative control. Furthermore, the epithelial-mesenchymal transition (EMT) was inhibited after LINC01234 knockdown. Additionally, LINC01234 knockdown impaired hypoxia-inducible factor-2a (HIF-2 $\alpha$ ) pathways $\square$ including a suppression of the expression of HIF- $2 \alpha$, vascular endothelial growth factor A (VEGFA), epidermal growth factor receptor (EGFR), c-Myc, Cyclin D1 and MET. Together, these datas showed that LINC01234 was likely to regulate the progression of CCRCC by HIF-2 $\alpha$ pathways, and LINC01234 was both a promising prognostic biomarker and a potential therapeutic target for $\mathrm{CCRCC}$. 


\section{Long Non-coding RNA LINC01234 Regulates}

\section{Proliferation, Migration and Invasion via HIF-2 $\alpha$}

\section{Pathways in Clear Cell Renal Cell Carcinoma Cells}

5

6

Feilong Yang ${ }^{1}$, Cheng Liu ${ }^{1}$, GuoJiang Zhao ${ }^{1}$, Liyuan Ge$^{1}$, Yimeng Song ${ }^{1}$, Zhigang Chen ${ }^{1}$, Zhuo Liu $^{1}$, Kai Hong ${ }^{1}$, Lulin Ma ${ }^{1}$

${ }^{1}$ Department of Urology, Peking University Third Hospital, Beijing, China

Corresponding Author:

Lulin $\mathrm{Ma}^{1}$

49 North Garden Road, Haidian District, Beijing 100191, China

Email address: malulin@medmail.com.cn

Kai Hong 1

49 North Garden Road, Haidian District, Beijing 100191, China

Email address: kenhong99@hotmail.com

\section{Abstract}

Long non-coding RNAs (lncRNAs) have been proved to have an important role in different malignancies including clear cell renal cell carcinoma (ccRCC). However, their role in disease progression is still not clear. The objective of the study was to identify lncRNA-based prognostic biomarkers and further to investigate the role of one lncRNA LINC01234 in progression of ccRCC cells. We found that six adverse prognostic lncRNA biomarkers including LINC01234 were identified in ccRCC patients by bioinformatics analysis using The Cancer Genome Atlas (TCGA) database. LINC01234 knockdown impaired cell proliferation, migration and invasion in vitro as compared to negative control. Furthermore, the epithelial-mesenchymal transition (EMT) was inhibited after LINC01234 knockdown. Additionally, LINC01234 knockdown impaired hypoxia-inducible factor-2a (HIF-2 $\alpha$ ) pathways, including a suppression of the expression of HIF-2 $\alpha$, vascular endothelial growth factor A (VEGFA), epidermal growth factor receptor (EGFR), c-Myc, Cyclin D1 and MET. Together, these datas showed that LINC01234 was likely 
33 to regulate the progression of ccRCC by HIF-2 $\alpha$ pathways, and LINC01234 was both a

34 promising prognostic biomarker and a potential therapeutic target for ccRCC.

\section{Introduction}

36

In 2018, it was predicted that 403,262 new cases would be diagnosed with kidney cancer in 185 countries and 175,098 cases would be dead (Bray et al. 2018). In 36 kinds of cancers, the morbidity and mortality of kidney cancer were $2.2 \%$ and $1.8 \%$ respectively (Bray et al. 2018). Clear cell RCC (ccRCC) is the most common subtype of RCC and it accounts for $75 \%$ (Song et al. 2018). Although surgery is still the preferred therapeutic option for the localized and locally advanced ccRCC, the long-term prognosis remains unsatisfactory and unpredictable. Current existed evaluation approach for prognosis of ccRCC is mainly based on clinicopathologic data, such as TNM staging. However, it does not reflect the biological heterogeneity of cancer (Cheng 2018). Therefore, there is an urgent need for discovering a new prognostic model and biomarkers for ccRCC. Moreover, it is necessary to understand the molecular mechanisms of the prognostic biomarkers underlying ccRCC development.

Long non-coding RNA (lncRNA) is a kind of RNA transcripts with a length of $>200$ nucleotides. Unlike mRNA, it does not encode proteins. Currently, it is reported that lncRNA is engaged in numerous important biological processes and the development and progression of numerous human diseases, including ccRCC (Esteller 2011; Gupta et al. 2010; Jin et al. 2017; Martens-Uzunova et al. 2014; Ponting et al. 2009; Quinn \& Chang 2016; Rinn \& Chang 2012). The overexpression, deficiency or mutation of IncRNA are associated with the tumor formation, progression, metastasis and prognosis in many human malignancies including ccRCC (Esteller 2011; Ghaffar et al. 2018; Gupta et al. 2010; He et al. 2018; Jin et al. 2017; Martens-Uzunova et al. 2014). However, the functions of the majority of lncRNA are not well understood. In our study, we constructed a lncRNA-based prognostic model and identified six lncRNAs as independent prognostic biomarkers in ccRCC, including LINC01234.

Several studies showed that LINC01234 was upregulated and had oncogenic potentials in several cancers, such as gastric cancer (Chen et al. 2018), esophageal cancer (Ghaffar et al. 2018), and colorectal adenocarcinoma (He et al. 2018). Most ccRCC are associated with loss of von HippelLindau tumor suppressor (pVHL) function and deregulation of hypoxia pathways (Schödel et al. 2016). Adaptation to hypoxia plays an important role in the progression of ccRCC (Garje et al. 2018). Hypoxia is mediated via hypoxia-inducible factors (HIFs) HIF- $1 \alpha$ and HIF-2 $\alpha$ (Semenza 2012). Recently, studies showed that HIF- $2 \alpha$, rather than HIF-1 $\alpha$, was a predominant driver in renal cancer progression (Keith et al. 2011). Although HIF-1 $\alpha$ can act as a ccRCC tumor suppressor, HIF-1 $\alpha$ activity is commonly diminished by chromosomal deletion in ccRCC (Schödel et al. 2016). Conversely, HIF-2 $\alpha$ has emerged as the key HIF isoform acting as an 
68

69

70

71

72

73

74

75

76

77

78

79

80

81

82

83

84

85

86

87

88

89

90

91

92

93

94

95

96

97

98

99

100

101

102

oncogene that is essential for ccRCC tumor progression (Meléndez-Rodríguez et al. 2018;

Schödel et al. 2016). The polymorphisms at the HIF-2 $\alpha$ gene locus predispose to the development of ccRCC, and HIF-2 $\alpha$ promotes tumor growth (Schödel et al. 2016). Indeed, preclinical and clinical data have shown that pharmacological inhibitors of HIF- $2 \alpha$ can efficiently inhibit ccRCC growth (Meléndez-Rodríguez et al. 2018). However, the role of LINC01234, as well as the relationship between LINC01234 and HIF-2 $\alpha$ in ccRCC remains unclear. In the present study, we showed that LINC01234 was likely to regulate the progression of ccRCC by HIF-2 $\alpha$ pathways. Therefore, LINC01234 might serve as a promising prognostic biomarker and a potential therapeutic target for patients with ccRCC.

\section{Materials \& Methods}

\section{LncRNA expression and clinical datasets of ccRCC cases}

The TCGA Research Network was available at http://cancergenome.nih.gov/ (Deng et al. 2016). The datasets for ccRCC cases within the TCGA database were downloaded using the GDC Data Portal. The version of the dataset was: Data Release 14.0-December 18, 2018.

\section{Differentially expression analysis to identify differentially expressed IncRNAs}

Differentially expression analysis was performed as previously described (Yang et al. 2019). A volcano plot was plotted for the differentially expressed lncRNAs.

\section{Univariate cox regression and least absolute shrinkage and selection operator (LASSO)} regression to identify key prognostic IncRNAs

The univariate cox regression was performed for the differentially expressed lncRNAs. Then, the statistically significant $\operatorname{lncRNAs}(\mathrm{p}<0.05)$ were used for LASSO regression to identify key prognostic lncRNAs. The univariate cox regression and LASSO regression were performed as previously described (Yang et al. 2019).

\section{Multivariate cox regression to establish the prognostic model}

The multivariate cox regression was performed for the key prognostic lncRNAs as previously described (Yang et al. 2019). It calculated the risk score for each patient. Based on the median of the risk score, all patients were divided into the high-risk group and low-risk group. A heatmap was plotted to present the expression levels of the key prognostic lncRNAs in the two groups. And a forest plot was plotted to present the hazard ratio (HR) and 95\% confidence interval (CI) for the key prognostic lncRNAs.

ROC curve and $C$-index to evaluate the prognostic model

The 3-year and 5-year time-dependent receiver operating characteristic (ROC) curves, the area under the ROC curves (AUCs) and the C-index were performed as previously described (Yang et al. 2019).

Kaplan-Meier (K-M) survival analysis to identify independent prognostic biomarkers 
103 The R package 'survival' (cran.r-project. org/web/packages/survival/index.html) was used for $104 \mathrm{~K}-\mathrm{M}$ survival analysis. Firstly, The K-M survival analysis was performed for the high-risk

105

106

107

108

109

110

111

112

113

114

115

116

117

118

119

120

121

122

123

124

125

126

127

128

129

130

131

132

133

134

135

136

137

138 group and the low-risk group. Then $\mathrm{K}-\mathrm{M}$ survival curves were plotted individually for each statistically significant lncRNA from the result of the multivariate cox regression. Validation of the expression and prognostic significance of the independent prognostic biomarkers

Gene Expression Profiling Interactive Analysis (GEPIA) server (Tang et al. 2017) is a newly developed interactive web server and has been running for three years. It was used for analyzing the RNA sequencing expression data computed by a standard processing pipeline. Therefore, we validated the expression levels and prognostic significance of the independent prognostic biomarkers in patients with ccRCC via GEPIA server according to their Ensembl ID.

Cell culture

Human RCC cells Caki-2 and A498 (Chinese Academy of Medical Sciences Shanghai Cell Bank) were cultured in RPMI 1640 medium (Gibco, USA) containing 10\% fetal bovine serum (FBS) (Gibco, USA), $100 \mathrm{U} / \mathrm{ml}$ penicillin (Sigma-Aldrich, St Louis, MO) and $100 \mu \mathrm{g} / \mathrm{ml}$ streptomycin (Sigma-Aldrich). All cells were routinely cultured in $5 \% \mathrm{CO} 2$ at $37^{\circ} \mathrm{C}$.

\section{Lentivirus-mediated shRNA transfection}

The recombinant lentivirus with short hairpin RNA (shRNA) and the corresponding control lentivirus were purchased from Genechem (Shanghai, China). Transfection in vitro was performed following the manufacturer's protocols. Stable shRNA-expressing colonies were selected using puromycin (Solarbio, Beijing, China). The target sequences of shRNA were as follows: 5' - CCTCGGTCTCAGTTTCTCCATTTAT-3' (shRNA) and 5'TTCTCCGAACGTGTCACGT-3' (control) respectively.

\section{RNA extraction, reverse transcription and real-time quantitative PCR (qPCR)}

RNA extraction and reverse transcription were performed as previously described (Wang et al. 2020). QPCR was performed using SYBR Green Realtime PCR Master Mix (TOYOBO, Osaka, Japan) in the QuantStudio 5 Real-Time PCR System (Thermo Fisher Scientific, USA). The PCR primers are shown in Supplementary Table S1. The relative expression levels of genes were calculated using the $2^{-\Delta \Delta \mathrm{Ct}}$ method relative to GAPDH.

\section{CCK-8 cell proliferation assay}

Cells stably expressing LINC01234 shRNA or control vector were plated into 96-well plates (2000 cells per well) and incubated at $37^{\circ} \mathrm{C}$ under $5 \% \mathrm{CO} 2$ for $1,2,3$ or 4 days respectively. Then CCK-8 solution (Dojindo, Japan) was added into the culture medium, and the optical density (OD) at $450 \mathrm{~nm}$ was measured with a Microplate Reader (Bio-Rad Laboratories Inc, Hercules, CA, USA) after incubation for $1.5 \mathrm{~h}$. Each group had five duplicates and the experiment was performed in triplicate. 


\section{Cell colony formation assay}

140 Cells stably expressing LINC01234 shRNA or control vector were plated into $10 \mathrm{~cm}$ culture dish 141 (1500 cells per dish) and incubated for 14 days. Wells were fixed with 4\% paraformaldehyde and 142 stained with $0.1 \%$ crystal violet. The cell colonies with $>50$ cells were counted. Each group had 143 three duplicates and the experiment was performed in triplicate.

\section{Transwell assays}

145 Transwell assays including migration assays and invasion assays were performed as previously

146 described (Wang et al. 2020). Each group had three duplicates and the experiment was

147 performed in triplicate.

\section{Western blots}

149 Western blots were performed as described (Liu et al. 2013; Yang et al. 2018). Total cellular 150 protein was extracted using RIPA buffer (Beyotime, Shanghai, China) with 1\% of $100 \mathrm{mM}$ 151 PMSF (Solarbio, Beijing, China). Protein concentration was quantified using a BCA Protein 152 Quantitative Kit (Beyotime, Shanghai, China). Briefly, $30 \mu \mathrm{g}$ of protein was resolved by $10 \%$ 153 SDS-PAGE, and transferred to a PVDF membrane (Millipore, Billerica, MA). The membrane was blocked with 5\% skim milk and then probed with rabbit or mouse anti-human primary antibodies respectively. Next, the membranes were incubated with corresponding HRPconjugated goat anti-rabbit or anti-mouse IgG (1:1000 dilution) (CST, Boston, USA) and detected with Western Blotting Luminol Reagent (Santa Cruz, CA, USA). The experiment was performed in triplicate. Epithelial-Mesenchymal Transition (EMT) Antibody Sampler Kit \#9782 (all 1:1000 dilution) (CST, Boston, USA) were used for western blots. Besides, mouse antihuman primary antibody HIF-1 $\alpha$ (Abcam, Cambridge, MA, USA), rabbit anti-human primary antibodies HIF-2 $\alpha$ (Abcam, Cambridge, MA, USA), VEGFA (Abcam, Cambridge, MA, USA), EGFR (Abcam, Cambridge, MA, USA), c-Myc (CST, Boston, USA), Cyclin D1 (CST, Boston, USA) and MET (CST, Boston, USA) (all 1:1000 dilution) were also detected.

164

165

166

167

168

169

170

171

172

173

174

\section{Statistical analysis}

For the datasets from TCGA database, the software Perl, R (version 3.4.4), RStudio 1.2.1335Windows 7+ (64-bit) and $\mathrm{R}$ packages were used for data integration, extraction, analysis and visualization. Briefly, the R package "edgeR" was utilized to screen differentially expressed genes $\left(\mathrm{FDR}<0.05\right.$ and $\left|\log _{2} \mathrm{FC}\right|>2$ ). The univariate cox regression and the Lasso regression were performed to identify key prognostic factors. The multivariate cox regression and K-M survival curve were performed to establish the risk score model and identify independent prognostic factors. ROC curve and C-index were performed to estimate the prognostic power of the risk score model. For the data about the function of LINC01234, SPSS 22.0 (IBM, USA) and GraphPad Prism 5.01 (GraphPad Software, USA) were used for statistical analyses. The data was expressed as mean \pm SD from at least three independent experiments. Cell proliferation abilities 
175 of CCK-8 assay were compared with two-way ANOVA. Cell colony, migration and invasion

176 levels, as well as qPCR data were compared using the Student's t-test. A p $<0.05$ was considered 177 statistically significant.

\section{Results}

179 Identification of differentially expressed IncRNAs and key prognostic IncRNAs in patients 180 with ccRCC

A total of 70 normal tissue samples and 541 cancer tissue samples from patients with ccRCC were collected. 11,368 lncRNAs were extracted from the transcriptome Profiling. Compared with the normal tissues, a total of 1541 lncRNAs were identified as differentially expressed lncRNAs in tumor tissues (FDR $<0.05$ and $|\operatorname{logFC}|>2)$, including 1075 upregulated $(\operatorname{logFC}>2)$ and 466 downregulated $(\operatorname{logFC}<-2) \operatorname{lncRNAs}$ (Figure 1A) (Supplementary Table S2). Preliminarily, a total of 323 statistically significant lncRNAs were considered to be related to the prognosis by the univariate cox regression (Supplementary Table S3). Next, through the LASSO regression, 13 lncRNAs were identified as key prognostic lncRNAs (Figure 1B, 1C), which were used for the further establishment of the risk score model by multivariate cox regression.

The median cutoff point of the risk scores calculated by multivariate cox regression was 0.842 . All patients were divided into the high-risk group and low-risk group. It was revealed that the patients in the high-risk group had a significantly worse overall survival rate than those in the low-risk group ( $<$ 0.001) (Figure 2A). The AUC was 0.753 (3-year ROC curve) and 0.784 (5-year ROC curve) respectively, and the C-index was 0.753 (Figure 2B). In addition, it also presented the relationship between the survival time and the risk score for patients (the death and the alive) (Figure 2C). Moreover, a heatmap was plotted to illustrate the expression levels of the key prognostic lncRNAs in the high-risk group and low-risk group (Figure 2D).

\section{Identification of independent prognostic biomarkers}

The multivariate cox regression revealed HR and 95\% CI for the 13 key prognostic lncRNAs with a forest plot (Figure 3). It indicated 6 statistically significant lncRNAs as the independent prognostic biomarkers, including lncRNAs AC009654.1, AC012615.3, AC092490.2, AL357507.1, LINC01234 and LINC01956. Moreover, K-M survival analysis was performed for the 6 lncRNAs. It revealed that all the 6 lncRNAs with high expression levels predicted a significantly worse overall survival rate than the low expressed one (Figure 4A-F). Therefore, they could serve as the adverse independent prognostic factors. Furthermore, we validated the expression levels and prognostic significance of the 6 lncRNAs in patients with ccRCC via GEPIA server. It suggested that AL357507.1, LINC01234, and LINC01956 were highly expressed at higher pathological stage of the disease, while LINC01234 exhibited the highest 
210 significance in terms of expression at different pathological stage of the disease (Figure 5A-F).

211 Moreover, GEPIA server revealed the significance of LINC01234 in terms of survival time. The

212 high expression level of LINC01234 predicted a significantly worse disease-free survival rare or 213 overall survival rate than the low expressed one (Figure 5G, 5H). Unfortunately, GEPIA server 214 could not provide the prognostic significance of the other 5 lncRNAs because the server showed 215 the sample size was insufficient.

216 LINC01234 knockdown suppressed the proliferation and clone formation of ccRCC cells

217 Knockdown of LINC01234 was performed in Caki-2 and A498 cells by the lentivirus-mediated 218 shRNA transfection. It suggested that the expression of LINC01234 was reduced in Caki-2 and 219 A498 cells, which was validated by qPCR (Figure 6A). Next, the CCK-8 assay revealed the 220 proliferations of Caki-2 and A498 cells were significantly suppressed (Figure 6B, 6C).

221 Moreover, cell colony formation assay was performed to analyze the role of LINC01234 in the 222 colony formation of Caki-2 and A498 cells. As shown in Figure 6D-I, the clonogenic capacities 223 of Caki-2 and A498 cells were dramatically inhibited. Obviously, it indicated that LINC01234 224 played an important role in the proliferation and colony formation of Caki-2 and A498 cells.

\section{LINC01234 depletion inhibited the migration and invasion of ccRCC cells}

226 The migration capabilities of Caki-2 and A498 cells were assessed by Transwell migration assay, 227 while the invasion capabilities of these cells were assessed by Transwell Matrigel invasion assay. 228 The results of the Transwell assay indicated that LINC01234 knockdown significantly inhibited 229 the migration capabilities of Caki-2 and A498 cells (Figure 7A-F). Similarly, the invasion 230 capabilities of Caki-2 and A498 cells were also suppressed following LINC01234 depletion 231 (Figure 7G-L). These findings demonstrated that LINC01234 played an important role in the 232 migration and invasion capacities of ccRCC cells.

\section{LINC01234 knockdown suppressed EMT process in ccRCC cells}

234 EMT process was closely related to the migration and invasion of cancer cells. Therefore, the 235 mRNA levels of EMT-associated genes and the levels of EMT-associated proteins were detected 236 by RT-PCR and western blots respectively. As shown in Figure 8A and 8B, we found that the 237 mRNA level of epithelial marker E-cadherin was increased, while the mRNA level of 238 mesenchymal marker N-cadherin was decreased in Caki-2 and A498 cells following LINC01234 239 knockdown. Similarly, as shown in Figure 8C, the protein expression levels of mesenchymal 240 markers Vimentin and N-cadherin were significantly decreased in Caki-2 and A498 cells with 241 LINC01234 knockdown, while the protein expression level of epithelial marker E-cadherin was 242 upregulated. Moreover, the protein expression level of the transcription factor Snail was 243 decreased in Caki-2 and A498 cells with LINC01234 knockdown. In addition, the protein 244 expression level of $\beta$-catenin was also inhibited in Caki-2 and A498 cells following LINC01234 245 depletion. 
246 LINC01234 suppression suppressed HIF-2 $\alpha$ pathways in ccRCC cells

247 As shown in Figure 8A and 8B, we found that the mRNA levels of HIF-2 $\alpha$ and vascular

248 endothelial growth factor A (VEGFA) were decreased in Caki-2 and A498 cells following

249 LINC01234 knockdown. Similarly, as shown in Figure 8D, we found that the protein expression

250 levels of HIF-1 $\alpha$ and HIF-2 $\alpha$ were significantly decreased in Caki-2 and A498 cells with

251 LINC01234 knockdown. Additionally, we found the protein expression levels of several target

252 genes of HIF-2 $\alpha$, including VEGFA, epidermal growth factor receptor (EGFR), c-myc, Cyclin

253 D1 and MET, were also inhibited in Caki-2 and A498 cells following LINC01234 depletion.

\section{Discussion}

255

256

257

258

259

260

261

262

263

264

265

266

267

268

269

270

271

272

273

274

275

276

277

278

279

280

LncRNA was a kind of long RNA transcripts (> 200 nucleotides) and it had no apparent proteincoding potentials (Quinn \& Chang 2016). Even so, IncRNA possessed a wide range of biological functions involved in multiple vital cellular activities (Shen et al. 2015). Generally, lncRNA achieved its function by regulating gene expression in the levels of epigenetics, transcription and post-transcription (Lee 2012; Wang \& Chang 2011). It could serve as a molecular signal, a molecular decoy, a molecular guide, or a molecular scaffold to achieve its functions (Wang \& Chang 2011). The function of lncRNA was associated with its subcellular localization (Wang \& Chang 2011). More specifically, lncRNA might be involved in chromatin regulation, gene transcription and alternative splicing of transcripts when it was in nucleus, while if it was in cytoplasm, it might serve as a competing endogenous RNA (ceRNA), and regulated the stability or translation of mRNA (Yang et al. 2019).

Recently, more and more evidences indicated that aberrations of lncRNA, such as overexpression, deficiency or mutation, played an important role in malignant phenotypes of cancers (Schmitt \& Chang 2016), including tumor formation, progression, metastasis and poor prognosis (Esteller 2011; Gupta et al. 2010; Martens-Uzunova et al. 2014; Yu et al. 2017; Yue et al. 2016). Some aberrant lncRNAs were also associated with lots of malignant biological behaviors of cancer cells, such as proliferation, apoptosis, migration and invasion (Ellinger et al. 2016; Huang et al. 2017; Yue et al. 2016). It was also reported that some aberrant lncRNAs could serve as prognostic indicators in ccRCC, such as lncRNA Fer1L4 (Cox et al. 2020). With the development of molecular biological techniques and bioinformatics, more and more lncRNAs were marked as novel biomarkers and prognostic signatures for ccRCC utilizing TCGA database. For example, lncRNA Fer1L4 was overexpressed in ccRCC tissues, and its high expression levels were found in higher grade, higher stage, and metastatic tumors (Cox et al. 2020). LncRNA Fer1L4 overexpression was also an independent prognostic factor for patients with ccRCC (Cox et al. 2020). It was also reported an 11-lncRNA signature (AC245100.1, AP002761.1, LINC00488, AC017033.1, LINC-PINT, COL5A1-AS1, AC026471.4, 
281 AL009181.1, LINC00524, HOTTIP, AL078590.3) and a 6-lncRNA signature (CTA-384D8.35, 282 CTD-2263F21.1, LINC01510, RP11-352G9.1, RP11-395B7.2, RP11-426C22.4) were clearly 283 linked to the overall survival (OS) rate of ccRCC patients based on TCGA database (Zeng et al. 284 2019; Zhang et al. 2019).

285 In the present study, utilizing the TCGA database, we identified 1541 differentially expressed 286 IncRNAs. More importantly, we not only constructed a 13-lncRNA-based risk score model with 287 moderate accuracy, but also identified 6 independent adverse prognostic lncRNAs for patients with ccRCC, including lncRNA AC009654.1, AC012615.3, AC092490.2, AL357507.1,

290

291

292

293

294

295

296

297

298

299

300

301

302

303

304

305

306

307

308

309

310

311

312

313

314

315

316 LINC01234 and LINC01956. It was similar to a recent study which suggested that lncRNA AC009654.1, AC092490.2, LINC00524, LINC01234 and LINC01885 were significantly associated with ccRCC prognosis (Zhang et al. 2020). The expression levels of the 6 lncRNAs above were upregulated in ccRCC tissues and the high expression levels of them predicted a worse overall survival in ccRCC patients. Furtherly, we investigated their expression levels at different pathological stages and validated their prognostic significance in ccRCC patients via GEPIA server. It revealed AL357507.1, LINC01234, and LINC01956 were highly expressed at higher pathological stages of the disease, while LINC01234 exhibited the highest significance in terms of expression at different pathological stages of the disease. It was a very interesting finding, because the pathological stage was closely associated with the prognosis of ccRCC patients. Moreover, GEPIA server revealed the significance of LINC01234 in terms of survival time. Unfortunately, GEPIA server could not provide the prognostic significance of the other 5 lncRNAs because the server showed the sample size was insufficient. Besides, I also referred to the recent studies and references about these six lncRNAs. Nevertheless, except limited researches for LINC01234, there are no investigations for them currently and it deserves to further researches. Therefore, we mainly focused on lncRNA LINC01234 for the subsequent experiments.

Recently, partial functions and mechanisms of LINC01234 (also known as LCAL84) were reported in cancers, such as gastric cancer (Chen et al. 2018), esophageal cancer (Ghaffar et al. 2018), and colorectal adenocarcinoma (He et al. 2018). LINC01234 was upregulated and had oncogenic potentials in esophageal carcinoma cells in vitro (Ghaffar et al. 2018; He et al. 2018). LINC01234 was significantly associated with the prognosis of colorectal adenocarcinoma and the malignant biological behaviors of esophageal carcinoma cells including proliferation, migration, invasion and apoptosis (Ghaffar et al. 2018; He et al. 2018). Besides, LINC01234 expression was significantly upregulated in gastric cancer tissues and was associated with larger tumor size, advanced TNM stage, lymph node metastasis, and shorter survival time (Chen et al. 2018). Moreover, LINC01234 could serve as ceRNA to regulate core-binding factor $\beta$ (CBFB) expression by sponging miR-204-5p to regulate the apoptosis, growth arrest and tumorigenesis in 
317 gastric cancer (Chen et al. 2018). In our study, we also explored the role of LINC01234 in 318 ccRCC. It indicated that LINC01234 expression was upregulated in ccRCC tissues. LINC01234

319 was expressed increasingly as the stage increased. The high expression level of LINC01234 320 predicted a significantly worse disease-free survival rate or overall survival rate than the low 321 expressed one for the patients with ccRCC. Besides, LINC01234 knockdown inhibited 322 proliferation, migration, invasion and epithelial-mesenchymal transition (EMT) process of 323 ccRCC cells. More importantly, LINC01234 knockdown impaired the expression of HIF-1a, 324 HIF-2a, VEGFA, EGFR, c-Myc, Cyclin D1 and MET in Caki-2 and A498 cells following 325 LINC01234 depletion.

326 EMT was considered as an essential process during development whereby epithelial cells 327 acquired mesenchymal, fibroblast-like characteristics and displayed reduced intracellular adhesion and increased motility (Aigner et al. 2007; Moreno-Bueno et al. 2008). EMT played a critical role in in the progression of primary tumors towards spread and metastasis, as well as the migration and invasion of malignant tumor cells (Gloushankova et al. 2018; Peinado et al. 2007; Yang et al. 2018). Recently, an increasing number of studies supported the role of lncRNAs in the regulation of tumor progression and metastasis through the regulation of EMT (Gugnoni \& Ciarrocchi 2019). In the carcinogenic progression, downregulation of cell-adhesion molecules like epithelial cadherins, occludins, claudins, certain cytokeratins, and ZO-1 together with the coordinated upregulation of mesenchymal cadherins, vimentin, fibronectin and $\beta 1$ and $\beta 3$ integrins, promoted loss of cell-cell adhesion and apico-basal polarity and acquisition of invasive and migratory capacity (Gugnoni \& Ciarrocchi 2019; Lu \& Kang 2019). A group of transcription factors including Snail, Slug, Twist, zinc finger E-box-binding homeobox 1 and 2 (ZEB1, ZEB2) were well known to regulated EMT process partially or completely (Gugnoni \& Ciarrocchi 2019; Yang et al. 2018). Therefore, we detected the mRNA levels of EMT-associated genes and the expression levels of EMT-associated proteins in ccRCC cells by qPCR and western blots respectively following LINC01234 knockdown. It revealed that the mRNA level of epithelial marker E-cadherin was increased, while the mRNA level of mesenchymal marker N-cadherin was decreased in Caki-2 and A498 cells following LINC01234 knockdown. The protein expression levels of the transcription factor Snail and the epithelial markers N-cadherin and Vimentin were reduced, while the protein expression level of the mesenchymal marker Ecadherin was up-regulated in A498 and Caki-2 cells with LINC01234 knockdown. These findings indicated that the function of LINC01234 was associated with EMT process. EMT was impaired after LINC01234 knockdown. In addition, we also found that inhibition of the $\beta$ catenin pathway contributed to the EMT impairment after LINC01234 depletion. All these evidences suggested that LINC01234 knockdown could inhibit the cell proliferation, migration and invasion, as well as EMT process in ccRCC. During EMT process, LINC01234 knockdown 
353

354

355

356

357

358

359

360

361

362

363

364

365

366

367

368

369

370

371

372

373

374

375

376

377

378

379

380

381

382

383

384

385

386

387

388

might suppress the expression of transcription factor Snail, and further stimulate the expression of E-cadherin, and inhibit the expressions of Vimentin and $\mathrm{N}$-cadherin, which might result in a inhibition of malignant biological behaviors of ccRCC cells, such as cell proliferation, migration and invasion.

Hypoxia could induce ccRCC cells to undergo EMT, angiogenesis and metastasis (MeléndezRodríguez et al. 2018; Zhang et al. 2017). Adaptation to a hypoxic environment played an important role in the progression of ccRCC (Garje et al. 2018). Hypoxia was mediated via hypoxia-inducible factors (Semenza 2012). Previously, HIF-1 $\alpha$ was supposed to be a key oncogenic factor, but recent evidence showed HIF-2 $\alpha$ was a predominant driver in renal cancer progression (Keith et al. 2011). Currently, HIF-1 $\alpha$ is supposed to be a ccRCC tumor suppressor, but the activity of HIF-1 $\alpha$ is commonly diminished by chromosomal deletion in ccRCC (Schödel et al. 2016). Conversely, HIF-2 $\alpha$ has emerged as an oncogene that is essential for ccRCC tumor progression (Meléndez-Rodríguez et al. 2018; Schödel et al. 2016). The polymorphisms at the HIF-2 $\alpha$ gene locus predispose to the development of ccRCC, and HIF-2 $\alpha$ can promote tumor growth (Schödel et al. 2016). Indeed, preclinical and clinical data have shown that pharmacological inhibitors of HIF-2 $\alpha$ can efficiently inhibit ccRCC growth (MeléndezRodríguez et al. 2018). HIF-2 $\alpha$ was found to be more sensitive to moderate hypoxia and showed more enduring expression in hypoxic conditions (Zhang et al. 2017). HIF-2 $\alpha$ could translocate to the nucleus and bind to the hypoxia response elements (Garje et al. 2018). This binding resulted in the expression of several target genes involved in angiogenesis, proliferation, migration and invasion of cancer cells, such as VEGFA, EGFR, c-Myc, Cyclin D1 and MET (Garje et al. 2018). VEGFA played an important role in the formation of blood vessels, which was closely associated with carcinogenesis (Shi et al. 2019). In ccRCC, as a well-known target of HIF-2 $\alpha$, VEGFA also played a vital role in angiogenesis and was a key target of anti-cancer therapeutic agents (Garje et al. 2018; Meléndez-Rodríguez et al. 2018). Besides, EGFR, c-Myc and Cyclin D1 were associated with ccRCC cell cycle and proliferation (Meléndez-Rodríguez et al. 2018). EGFR signaling could also promote ccRCC survival (Meléndez-Rodríguez et al. 2018). Moreover, MET was related to ccRCC metastasis (Meléndez-Rodríguez et al. 2018). Based on this above, we detected HIF-2 $\alpha$ pathways after LINC01234 depletion. It revealed that the mRNA levels of HIF-2 $\alpha$ and VEGFA were decreased in A498 and Caki-2 cells with LINC01234 knockdown. Similarly, the expression levels of proteins HIF-2 $\alpha$, VEGFA, EGFR, c-Myc, Cyclin D1 and MET were reduced in A498 and Caki-2 cells with LINC01234 knockdown. In our study, LINC01234 was expressed increasingly as the stage increased and its high expression level predicted a significantly worse disease-free survival rate or overall survival rate for the patients with ccRCC. LINC01234 knockdown suppressed cell proliferation, migration and invasion of ccRCC cells. Combined with all these findings above, it suggested that LINC01234 knockdown 
389 might suppress the expression of HIF-2 $\alpha$, and then inhibit the expression of VEGFA, EGFR, c390 Myc, Cyclin D1 and MET, which might further inhibit the proliferation, metastasis and survival 391 of ccRCC.

392 Unfortunately, there was several limitations in our study. Firstly, the function of lncRNA was 393 associated with its subcellular localization, but we did not identify the subcellular localization of 394 LINC01234 in ccRCC cell lines. Secondly, although LINC01234 functioned as ceRNA to regulate 395 CBFB expression by sponging miR-204-5p in gastric cancer, we did not identify any miRNAs as 396 direct targets of LINC01234 to investigate whether LINC01234 was a ceRNA for miRNAs in 397 ccRCC. It deserves to more investigations.

\section{Conclusions}

399 In summary, we constructed a lncRNA-based prognostic model with moderate accuracy and 400 identified LINC01234 as an independent prognostic biomarker in ccRCC. Moreover, 401 LINC01234 knockdown might inhibit the proliferation and metastasis of ccRCC cells by 402 suppressing HIF-2 $\alpha$ pathways. Therefore, LINC01234 might serve as a promising prognostic 403 biomarker and a potential therapeutic target for patients with ccRCC.

404

405

406

407

408

409

410

411

412

413

414

415

416

417

418

419

420

421

\section{Acknowledgements}

We specially gratitude to The Cancer Genome Atlas (TCGA) project (https://portal.gdc.cancer.gov/) for its valuable public data set.

\section{References}

Aigner K, Dampier B, Descovich L, Mikula M, Sultan A, Schreiber M, Mikulits W, Brabletz T, Strand D, Obrist P, Sommergruber W, Schweifer N, Wernitznig A, Beug $H$, Foisner R, and Eger A. 2007. The transcription factor ZEB1 (deltaEF1) promotes tumour cell dedifferentiation by repressing master regulators of epithelial polarity. Oncogene 26:6979-6988. 10.1038/sj.onc.1210508

Bray F, Ferlay J, Soerjomataram I, Siegel RL, Torre LA, and Jemal A. 2018. Global cancer statistics 2018: GLOBOCAN estimates of incidence and mortality worldwide for 36 cancers in 185 countries. CA Cancer J Clin 68:394-424. 10.3322/caac.21492

Chen X, Chen Z, Yu S, Nie F, Yan S, Ma P, Chen Q, Wei C, Fu H, Xu T, Ren S, Sun M, and Wang Z. 2018. Long Noncoding RNA LINC01234 Functions as a Competing Endogenous RNA to Regulate CBFB Expression by Sponging miR-204-5p in Gastric Cancer. Clin Cancer Res 24:2002-2014. 10.1158/1078-0432.ccr-17-2376

Cheng P. 2018. A prognostic 3-long noncoding RNA signature for patients with gastric cancer. $J$ Cell Biochem 119:9261-9269. 10.1002/jcb.27195 
422

423

424

425

426

427

428

429

430

431

432

433

434

435

436

437

438

439

440

441

442

443

444

445

446

447

448

449

450

451

452

453

454

455

456

Cox A, Tolkach Y, Kristiansen G, Ritter M, and Ellinger J. 2020. The IncRNA Fer1L4 is an adverse prognostic parameter in clear-cell renal-cell carcinoma. Clin Transl Oncol. 10.1007/s12094-020-02291-0

Deng M, Bragelmann J, Schultze JL, and Perner S. 2016. Web-TCGA: an online platform for integrated analysis of molecular cancer data sets. BMC Bioinformatics 17:72. 10.1186/s12859-016-0917-9

Ellinger J, Gevensleben H, Muller SC, and Dietrich D. 2016. The emerging role of non-coding circulating RNA as a biomarker in renal cell carcinoma. Expert Rev Mol Diagn 16:10591065. 10.1080/14737159.2016.1239531

Esteller M. 2011. Non-coding RNAs in human disease. Nat Rev Genet 12:861-874. 10.1038/nrg3074

Garje R, An JJ, Sanchez K, Greco A, Stolwijk J, Devor E, Rustum Y, and Zakharia Y. 2018. Current Landscape and the Potential Role of Hypoxia-Inducible Factors and Selenium in Clear Cell Renal Cell Carcinoma Treatment. Int J Mol Sci 19. 10.3390/ijms19123834

Ghaffar M, Khodahemmati S, Li J, Shahzad M, Wang M, Wang Y, Li C, Chen S, and Zeng Y. 2018. Long Non-coding RNA LINC01234 Regulates Proliferation, Invasion and Apoptosis in Esophageal Cancer Cells. J Cancer 9:4242-4249. 10.7150/jca.26095

Gloushankova NA, Zhitnyak IY, and Rubtsova SN. 2018. Role of Epithelial-Mesenchymal Transition in Tumor Progression. Biochemistry (Mosc) 83:1469-1476. $10.1134 / \mathrm{s} 0006297918120052$

Gugnoni M, and Ciarrocchi A. 2019. Long Noncoding RNA and Epithelial Mesenchymal Transition in Cancer. Int J Mol Sci 20. 10.3390/ijms20081924

Gupta RA, Shah N, Wang KC, Kim J, Horlings HM, Wong DJ, Tsai MC, Hung T, Argani P, Rinn JL, Wang Y, Brzoska P, Kong B, Li R, West RB, van de Vijver MJ, Sukumar S, and Chang HY. 2010. Long non-coding RNA HOTAIR reprograms chromatin state to promote cancer metastasis. Nature 464:1071-1076. 10.1038/nature08975

He Z, Dang J, Song A, Cui X, Ma Z, and Zhang Z. 2018. Identification of LINC01234 and MIR210HG as novel prognostic signature for colorectal adenocarcinoma. J Cell Physiol. 10.1002/jcp.27424

Huang HW, Xie H, Ma X, Zhao F, and Gao Y. 2017. Upregulation of LncRNA PANDAR predicts poor prognosis and promotes cell proliferation in cervical cancer. Eur Rev Med Pharmacol Sci 21:4529-4535.

Jin Y, Feng SJ, Qiu S, Shao N, and Zheng JH. 2017. LncRNA MALAT1 promotes proliferation and metastasis in epithelial ovarian cancer via the PI3K-AKT pathway. Eur Rev Med Pharmacol Sci 21:3176-3184. 
457 Keith B, Johnson RS, and Simon MC. 2011. HIF1alpha and HIF2alpha: sibling rivalry in hypoxic

458

459

460

461

462

463

464

465

466

467

468

469

470

471

472

473

474

475

476

477

478

479

480

481

482

483

484

485

486

487

488

489

490

491

492 tumour growth and progression. Nat Rev Cancer 12:9-22. 10.1038/nrc3183

Lee JT. 2012. Epigenetic regulation by long noncoding RNAs. Science 338:1435-1439. 10.1126/science. 1231776

Liu C, Wang C, Wang K, Liu L, Shen Q, Yan K, Sun X, Chen J, Liu J, Ren H, Liu H, Xu Z, Hu S, $\mathrm{Xu}$ D, and Fan Y. 2013. SMYD3 as an oncogenic driver in prostate cancer by stimulation of androgen receptor transcription. J Natl Cancer Inst 105:1719-1728. 10.1093/jnci/djt304

Lu W, and Kang Y. 2019. Epithelial-Mesenchymal Plasticity in Cancer Progression and Metastasis. Dev Cell 49:361-374. 10.1016/j.devcel.2019.04.010

Martens-Uzunova ES, Bottcher R, Croce CM, Jenster G, Visakorpi T, and Calin GA. 2014. Long noncoding RNA in prostate, bladder, and kidney cancer. Eur Urol 65:1140-1151. 10.1016/j.eururo.2013.12.003

Meléndez-Rodríguez F, Roche O, Sanchez-Prieto R, and Aragones J. 2018. Hypoxia-Inducible Factor 2-Dependent Pathways Driving Von Hippel-Lindau-Deficient Renal Cancer. Front Oncol 8:214. 10.3389/fonc.2018.00214

Moreno-Bueno G, Portillo F, and Cano A. 2008. Transcriptional regulation of cell polarity in EMT and cancer. Oncogene 27:6958-6969. 10.1038/onc.2008.346

Peinado H, Olmeda D, and Cano A. 2007. Snail, Zeb and bHLH factors in tumour progression: an alliance against the epithelial phenotype? Nat Rev Cancer 7:415-428. $10.1038 / \mathrm{nrc} 2131$

Ponting CP, Oliver PL, and Reik W. 2009. Evolution and functions of long noncoding RNAs. Cell 136:629-641. 10.1016/j.cell.2009.02.006

Quinn JJ, and Chang HY. 2016. Unique features of long non-coding RNA biogenesis and function. Nat Rev Genet 17:47-62. 10.1038/nrg.2015.10

Rinn JL, and Chang HY. 2012. Genome regulation by long noncoding RNAs. Annu Rev Biochem 81:145-166. 10.1146/annurev-biochem-051410-092902

Schmitt AM, and Chang HY. 2016. Long Noncoding RNAs in Cancer Pathways. Cancer Cell 29:452-463. 10.1016/j.ccell.2016.03.010

Schödel J, Grampp S, Maher ER, Moch H, Ratcliffe PJ, Russo P, and Mole DR. 2016. Hypoxia, Hypoxia-inducible Transcription Factors, and Renal Cancer. Eur Urol 69:646-657. 10.1016/j.eururo.2015.08.007

Semenza GL. 2012. Hypoxia-inducible factors: mediators of cancer progression and targets for cancer therapy. Trends Pharmacol Sci 33:207-214. 10.1016/j.tips.2012.01.005

Shen Y, Wang Z, Loo LW, Ni Y, Jia W, Fei P, Risch HA, Katsaros D, and Yu H. 2015. LINC00472 expression is regulated by promoter methylation and associated with 
493

494

495

496

497

498

499

500

501

502

503

504

505

506

507

508

509

510

511

512

513

514

515

516

517

518

519

520

521

522

523

524

525

526

527

528

disease-free survival in patients with grade 2 breast cancer. Breast Cancer Res Treat 154:473-482. 10.1007/s10549-015-3632-8

Shi J, Zhang D, Zhong Z, and Zhang W. 2019. IncRNA ROR promotes the progression of renal cell carcinoma through the miR-206/VEGF axis. Mol Med Rep 20:3782-3792. 10.3892/mmr.2019.10636

Song E, Song W, Ren M, Xing L, Ni W, Li Y, Gong M, Zhao M, Ma X, Zhang X, and An R. 2018. Identification of potential crucial genes associated with carcinogenesis of clear cell renal cell carcinoma. J Cell Biochem 119:5163-5174. 10.1002/jcb.26543

Tang Z, Li C, Kang B, Gao G, Li C, and Zhang Z. 2017. GEPIA: a web server for cancer and normal gene expression profiling and interactive analyses. Nucleic Acids Res 45:W98w102. 10.1093/nar/gkx247

Wang G, Huang Y, Yang F, Tian X, Wang K, Liu L, Fan Y, Li X, Li L, Shi B, Hao Y, Xia C, Nie Q, Xin Y, Shi Z, Ma L, Xu D, and Liu C. 2020. High expression of SMYD3 indicates poor survival outcome and promotes tumour progression through an IGF-1R/AKT/E2F-1 positive feedback loop in bladder cancer. Aging (Albany NY) 12:2030-2048. 10.18632/aging. 102718

Wang KC, and Chang HY. 2011. Molecular mechanisms of long noncoding RNAs. Mol Cell 43:904-914. 10.1016/j.molcel.2011.08.018

Yang F, Song Y, Ge L, Zhao G, Liu C, and Ma L. 2019. Long non-coding RNAs as prognostic biomarkers in papillary renal cell carcinoma. Oncol Lett 18:3691-3697. 10.3892/ol.2019.10684

Yang F, Yu N, Wang H, Zhang C, Zhang Z, Li Y, Li D, Yan L, Liu H, and Xu Z. 2018. Downregulated expression of hepatoma-derived growth factor inhibits migration and invasion of prostate cancer cells by suppressing epithelial-mesenchymal transition and MMP2, MMP9. PLoS One 13:e0190725. 10.1371/journal.pone.0190725

Yu Y, Yang J, Li Q, Xu B, Lian Y, and Miao L. 2017. LINC00152: A pivotal oncogenic long noncoding RNA in human cancers. Cell Prolif 50. 10.1111/cpr.12349

Yue B, Qiu S, Zhao S, Liu C, Zhang D, Yu F, Peng Z, and Yan D. 2016. LncRNA-ATB mediated E-cadherin repression promotes the progression of colon cancer and predicts poor prognosis. J Gastroenterol Hepatol 31:595-603. 10.1111/jgh.13206

Zeng JH, Lu W, Liang L, Chen G, Lan HH, Liang XY, and Zhu X. 2019. Prognosis of clear cell renal cell carcinoma (ccRCC) based on a six-IncRNA-based risk score: an investigation based on RNA-sequencing data. J Transl Med 17:281. 10.1186/s12967-019-2032-y

Zhang C, Huang D, Liu A, Xu Y, Na R, and Xu D. 2020. Genome-wide screening and cohorts validation identifying novel IncRNAs as prognostic biomarkers for clear cell renal cell carcinoma. J Cell Biochem 121:2559-2570. 10.1002/jcb.29478

Peer) reviewing PDF | (2020:05:49159:1:1:NEW 26 Aug 2020) 
529 Zhang J, Zhang X, Piao C, Bi J, Zhang Z, Li Z, and Kong C. 2019. A long non-coding RNA

530

531

532

533

534 signature to improve prognostic prediction in clear cell renal cell carcinoma. Biomed Pharmacother 118:109079. 10.1016/j.biopha.2019.109079

Zhang Q, Lou Y, Zhang J, Fu Q, Wei T, Sun X, Chen Q, Yang J, Bai X, and Liang T. 2017. Hypoxia-inducible factor-2 $\alpha$ promotes tumor progression and has crosstalk with Wnt/ $\beta$ catenin signaling in pancreatic cancer. Mol Cancer 16:119. 10.1186/s12943-017-0689-5 


\section{Figure 1}

Identification of differentially expressed IncRNAs and key prognostic IncRNAs in patients with CCRCC.

(A) Identification of differentially expressed IncRNAs. A total of 11,368 IncRNAs were extracted from the transcriptome profiling and 1541 InCRNAs were identified as differentially expressed IncRNAs in tumor tissues, including 1075 upregulated (logFC > 2) and 466 downregulated (logFC < -2) IncRNAs. (B, C) Tuning parameter and variable selection by LASSO regression to identify key prognostic IncRNAs. A total of 323 significant IncRNAs were preliminarily associated with prognosis by the univariate cox regression, and finally 13 key prognostic InCRNAs were identified by LASSO regression. The numbers on the top of the figures indicated the number of the candidate IncRNAs for the corresponding lambda ( $\lambda$ ) value in LASSO regression. InCRNA, long non-coding RNA; FC, fold change; LASSO, least absolute shrinkage and selection operator. 

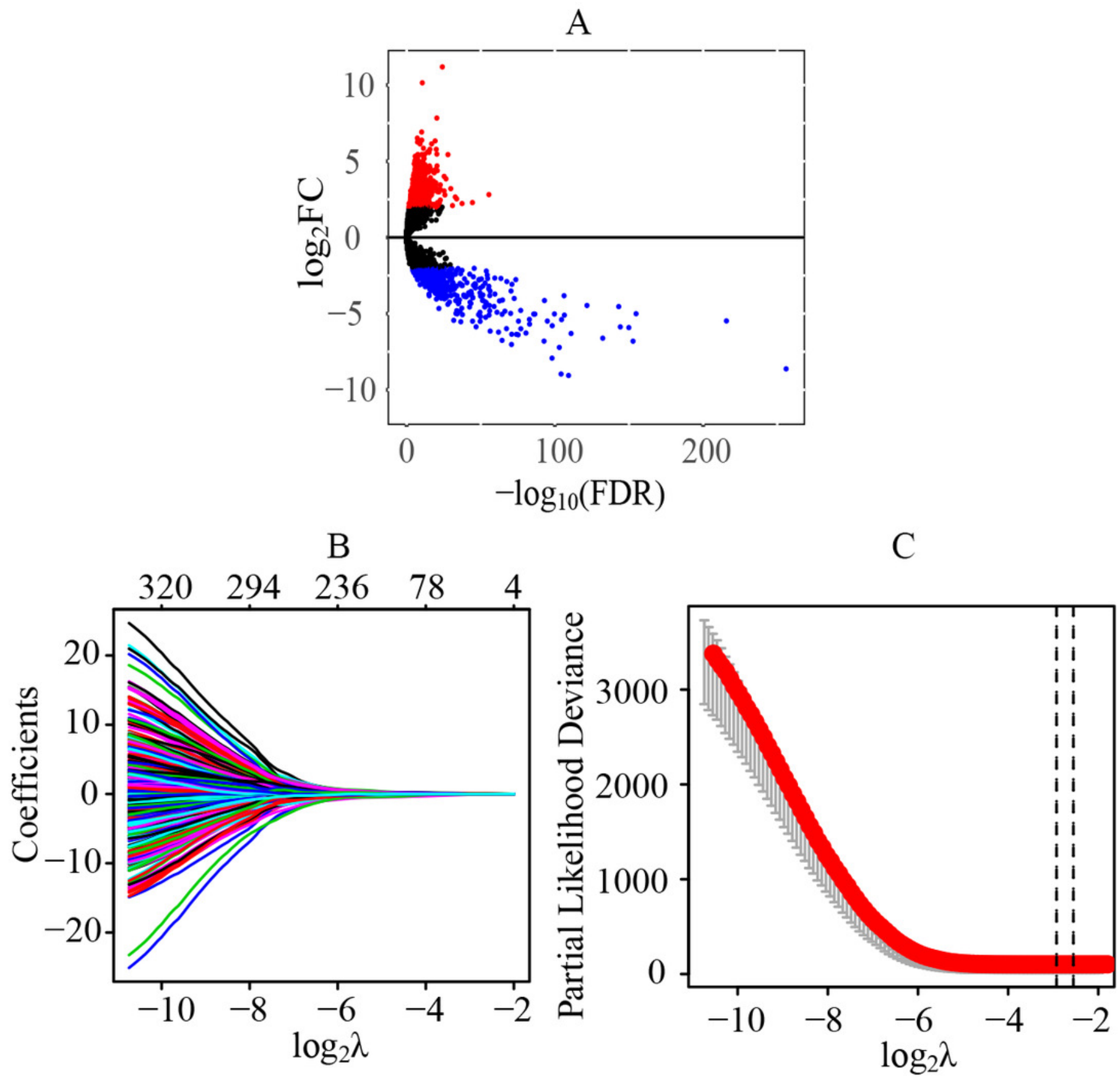


\section{Figure 2}

Establishment and evaluation of the prognostic model.

(A) K-M survival curve of the risk level. By multivariate cox regression based on the 13 key prognostic IncRNAs, the prognostic model was established and the patients were divided into high-risk and low-risk group. It revealed that the patients in the high-risk group had significantly worse OS rate than that of the low-risk group $(p<0.05)$. (B) 3-year and 5-year $R O C$ curves and C-index of the 13 key prognostic InCRNAs. $A \cup C=0.0 .753$ (3-year ROC) and $A U C=0.784$ (5-year ROC), C-index $=0.753$. (C) The distribution landscape of the alive and the dead of $c C R C C$ cases in the coordinate system of risk score and survival time. (D) Heatmap of the expression levels of the 13 key prognostic IncRNAs in the high-risk group and low-risk group. The red and blue represent increased and decreased normalized expression value of the IncRNAs in all patients, respectively. K-M, Kaplan-Meier; ROC, receiver operating characteristic; AUC, area under curve; InCRNAs, long non-coding RNAs; OS, overall survival; CCRCC, clear cell renal cell carcinoma. 
A

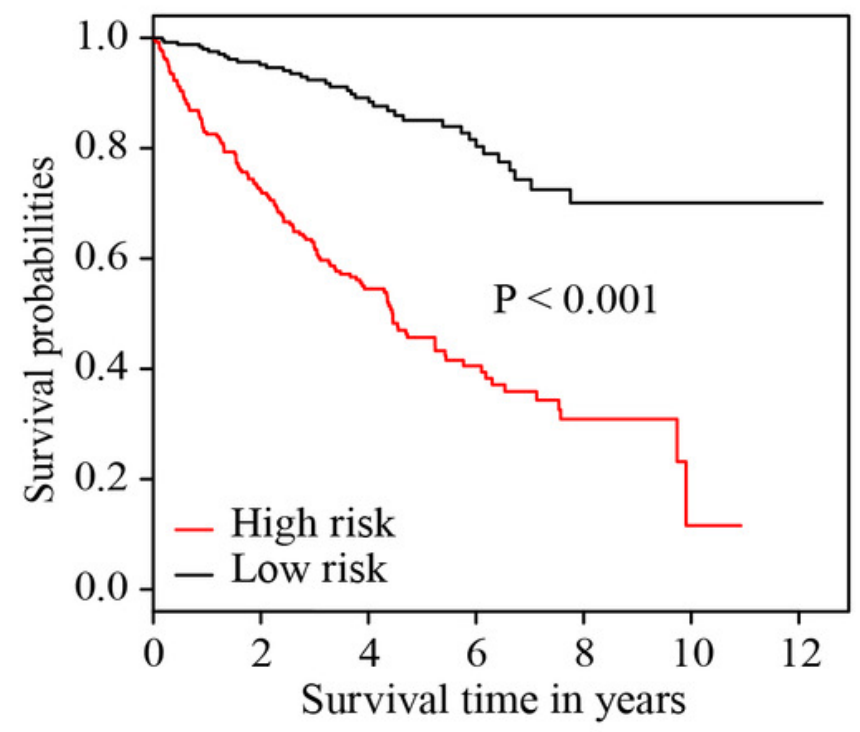

C

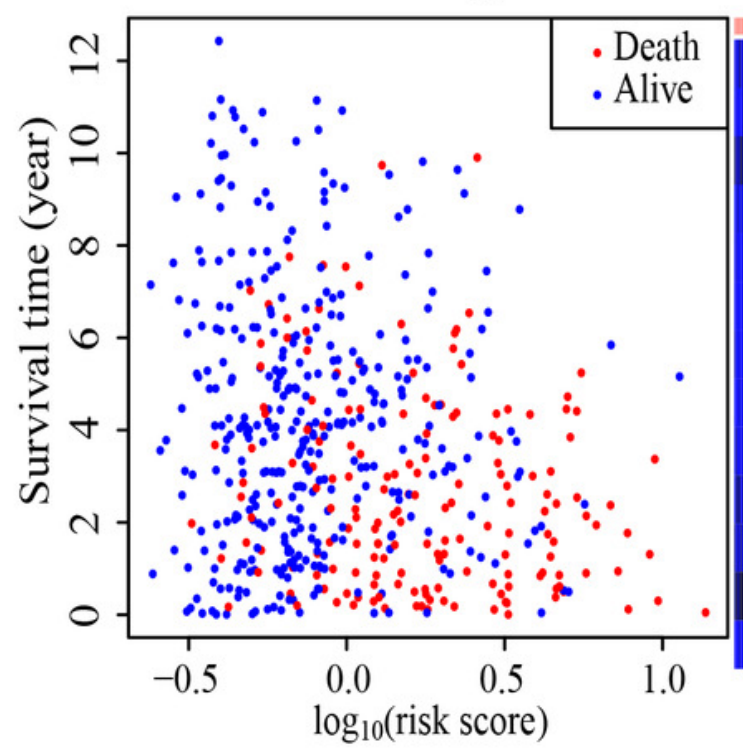

B

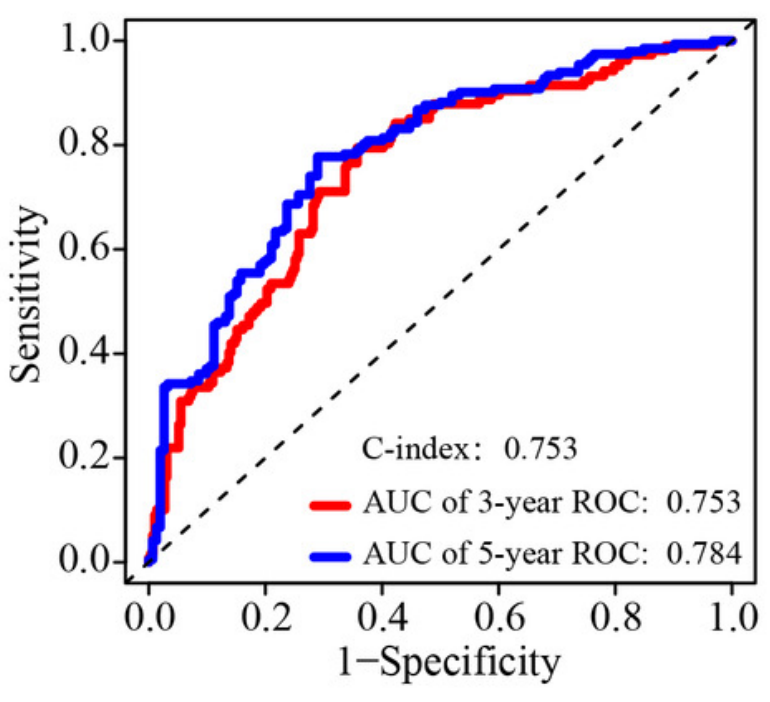

D

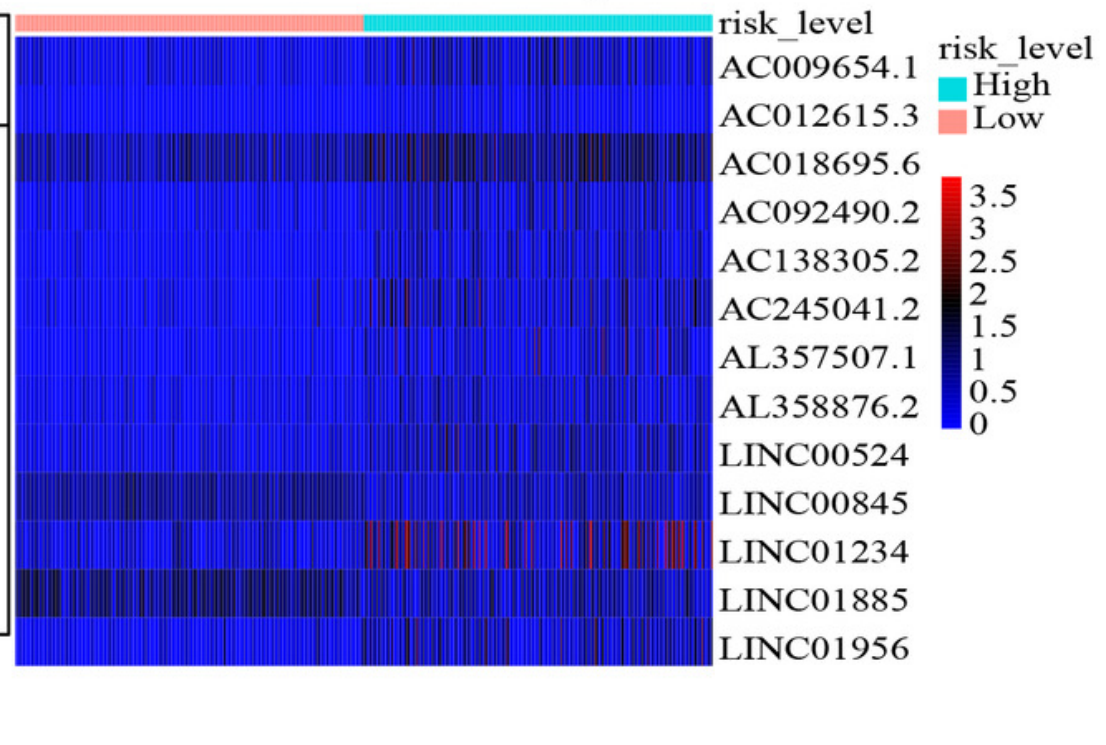




\section{Figure 3}

$H R$ and $95 \% \mathrm{Cl}$ of the 13 key prognostic IncRNAs by multivariate cox regression.

A total of 528 patients were included in the analysis. Of the 13 key prognostic IncRNAs, six had a significant influence on the OS rate of CCRCC patients, including AC009654.1, AC012615.3, AC092490.2, AL357507.1, LINC01234 and LINC01956 (all p < 0.05). HR, hazard ratio; $\mathrm{Cl}$, confidence interval; InCRNA, long non-coding RNA; $C C R C C$, clear cell renal cell carcinoma; OS, overall survival. 


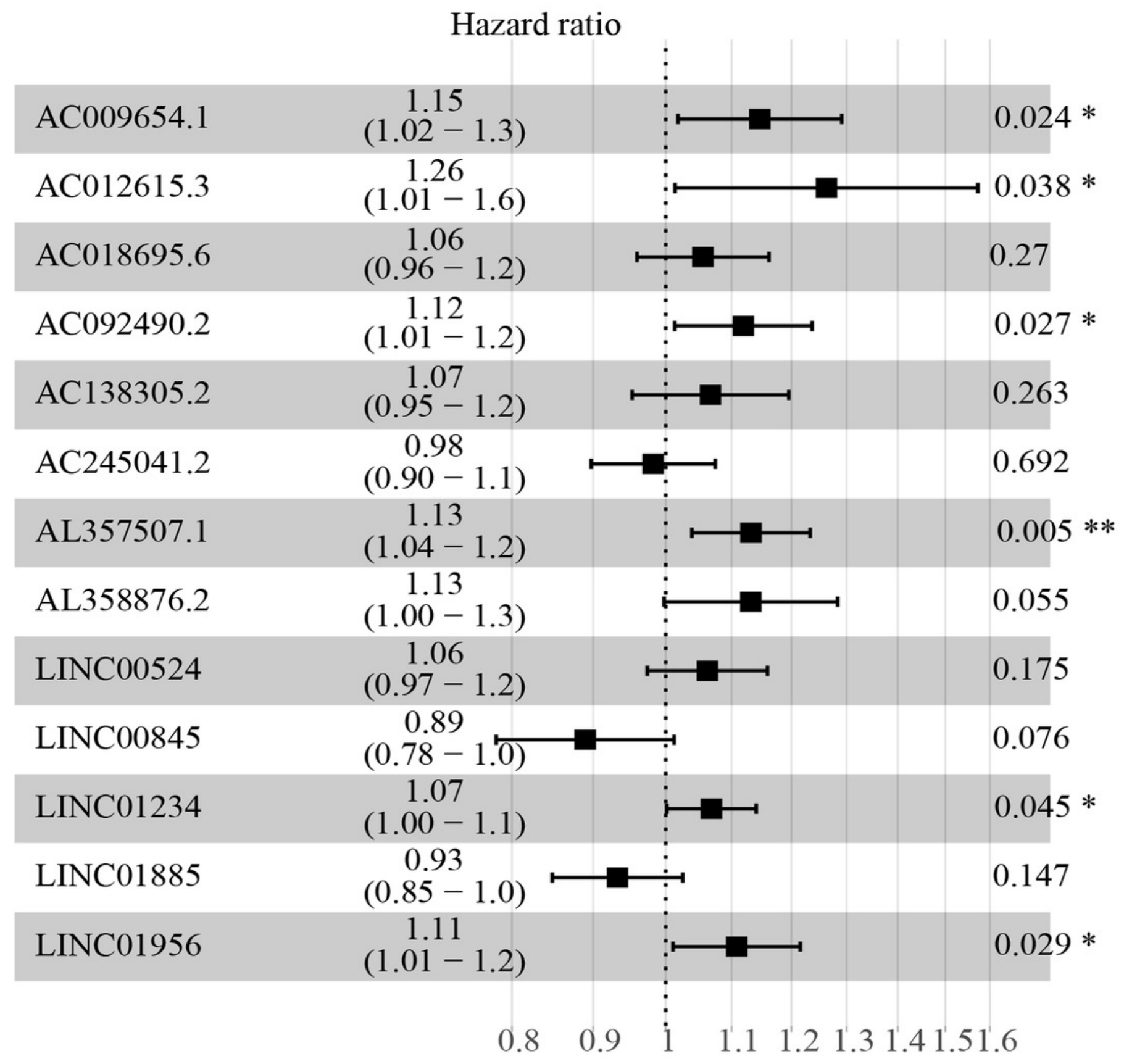


Figure 4

K-M survival curves of the six independent prognostic InCRNAs identified by multivariate cox regression.

It showed that the high expression levels of the IncRNAs, including AC009654.1 (A), AC012615.3 (B), AC092490.2 (C), AL357507.1 (D), LINC01234 (E) and LINC01956 (F), were correlated with the worse OS rate of patients with cCRCC (all $p<0.05$ ). K-M, Kaplan-Meier; OS, overall survival; cCRCC, clear cell renal cell carcinoma; IncRNA, long non-coding RNA.

A

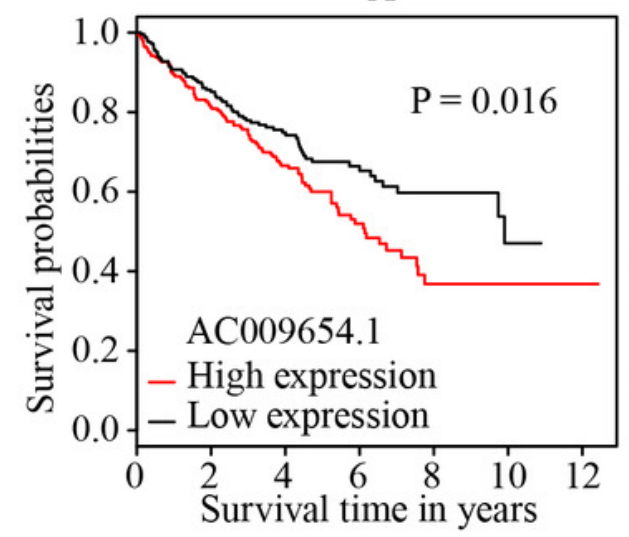

$\mathrm{D}$

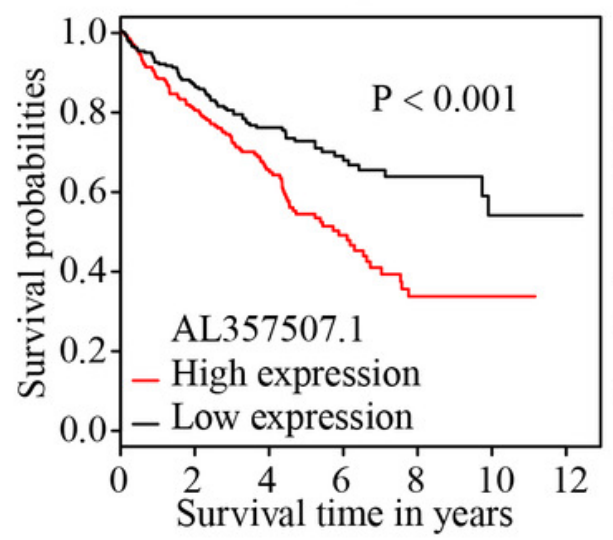

B

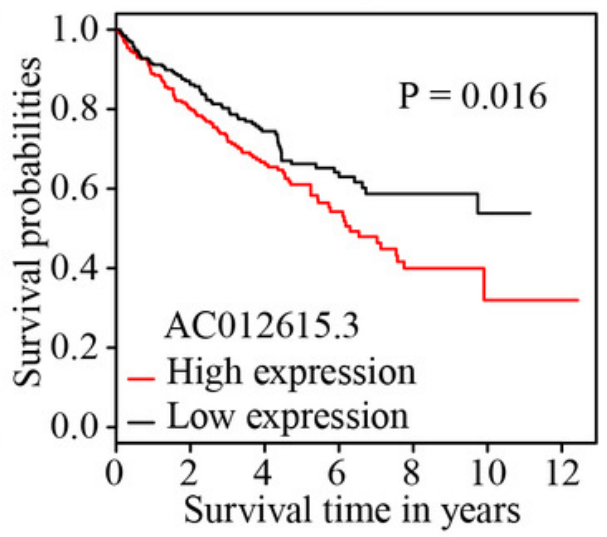

E

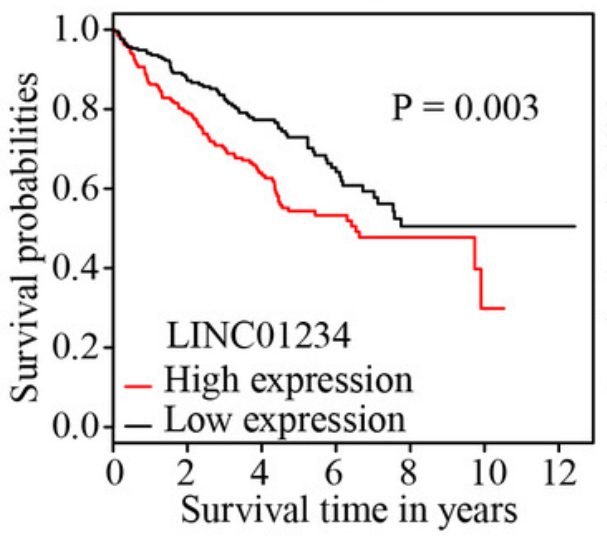

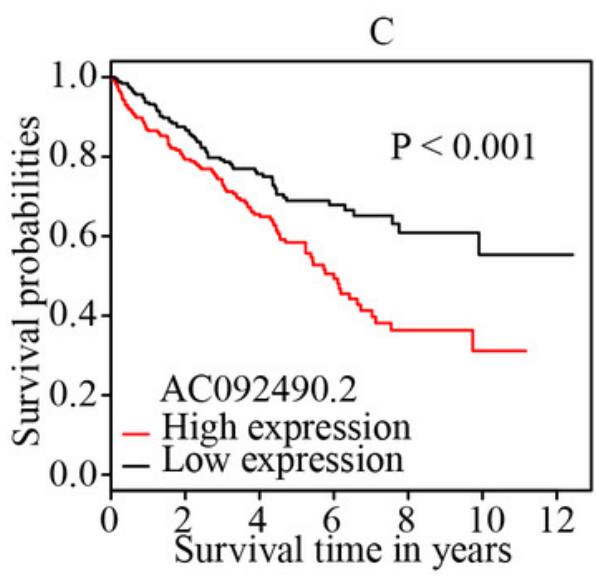

F

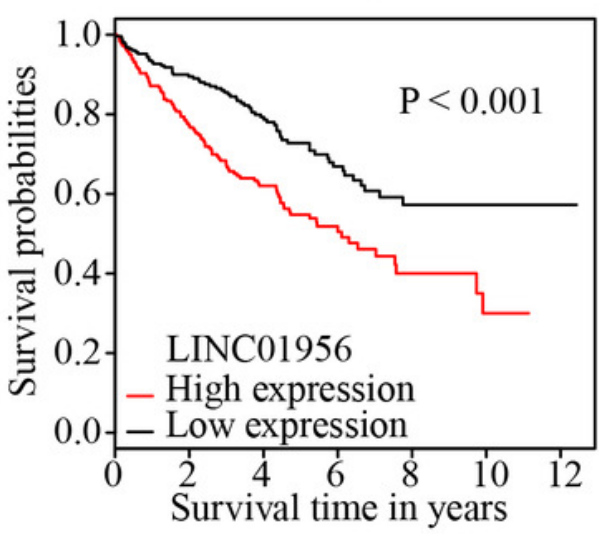




\section{Figure 5}

Validation of the expression and prognostic significance of the six independent prognostic InCRNAs by GEPIA server.

(A-F) Expression levels of the 6 independent prognostic InCRNAs in different stages of patients with cCRCC. It suggested that AL357507.1, LINC01234, and LINC01956 were highly expressed at higher pathological stage of the disease, while LINC01234 exhibited the highest significance in terms of expression levels at different pathological stage of the disease. $(G, H)$ Prognostic significance of LINC01234 in patients with cCRCC. It showed the high expression level of LINC01234 predicted a significantly worse disease-free survival rate or overall survival rate than that of the low expressed (all $p<0.05$ ). $C C R C C$, clear cell renal cell carcinoma; GEPIA, Gene Expression Profiling Interactive Analysis. 
A

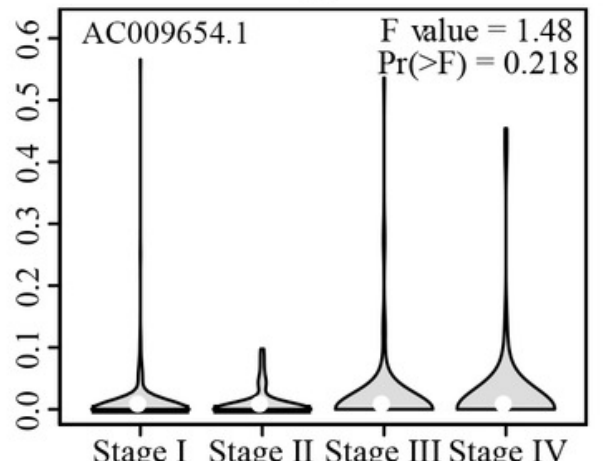

D

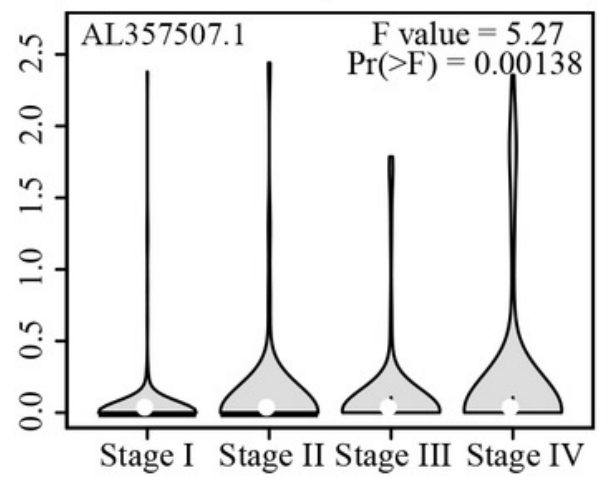

B

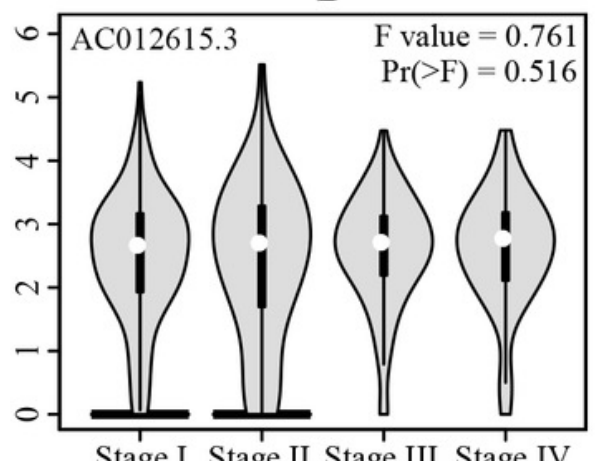

Stage I Stage II Stage III Stage IV

E

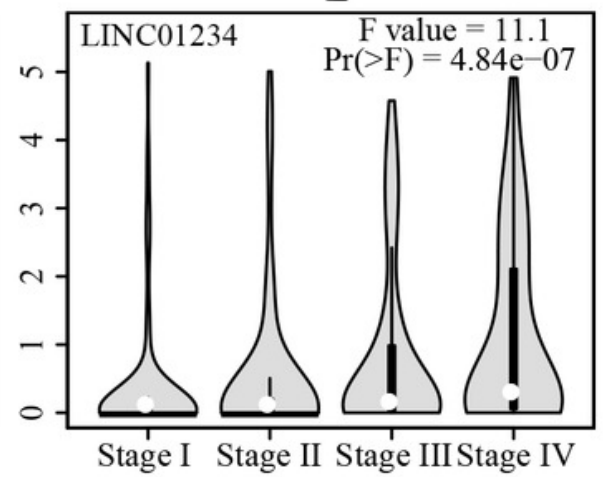

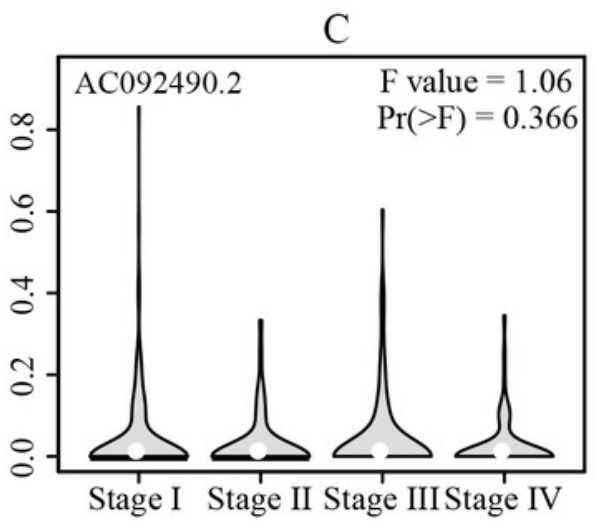

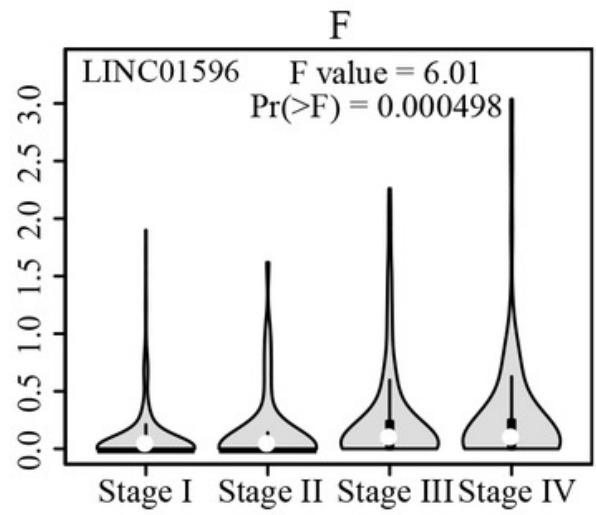

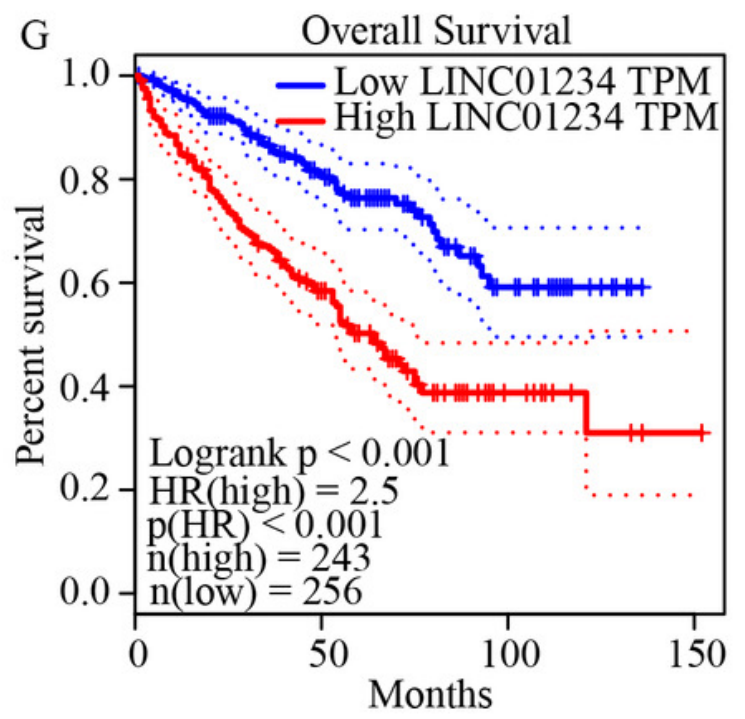

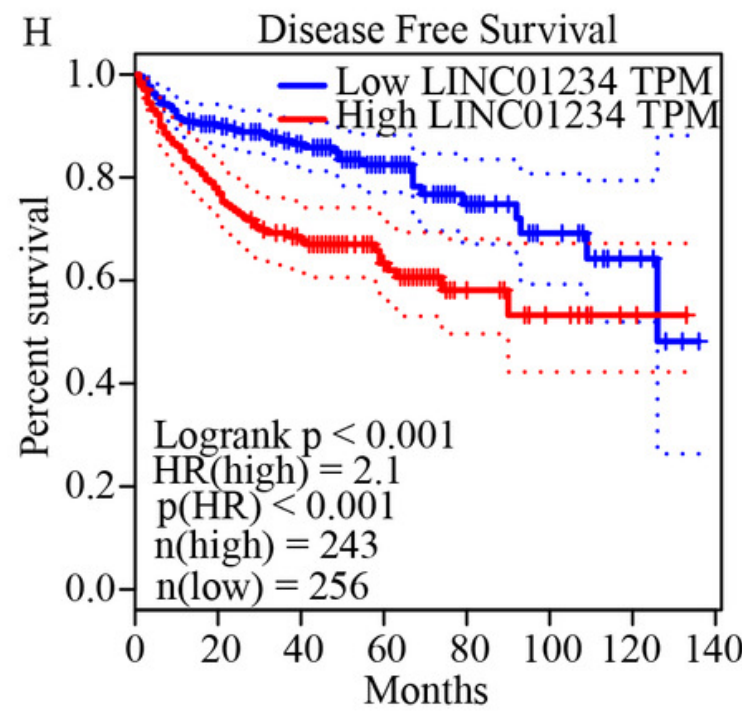




\section{Figure 6}

LINC01234 knockdown suppressed the proliferation and colony formation of CCRCC cells.

(A) QPCR analysis verified that LINC01234 was down-regulated in Caki-2 and A498 cells (all $p$ $<0.05)$. (B, C) CCK-8 assays showed that LINC01234 knockdown reduced the proliferation of Caki-2 and A498 cells respectively (all $p<0.05)$. ( $D-I)$ Colony formation assays showed that LINC01234 knockdown reduced the colony formation capabilities of Caki-2 and A498 cells respectively (all $p<0.05$ ). *, $p<0.05$; **, $p<0.01$; ***, $p<0.001$; ****, $p<0.0001$; cCRCC, clear cell renal cell carcinoma. 


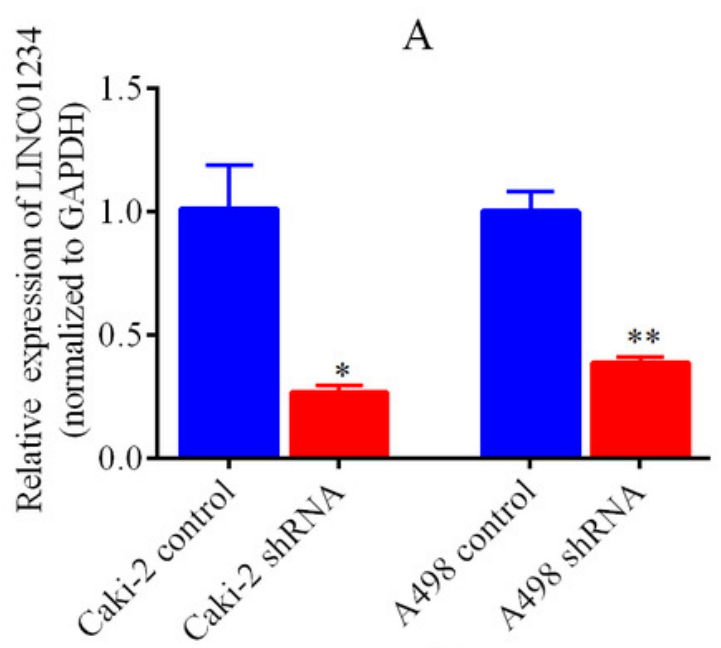

D

Caki-2

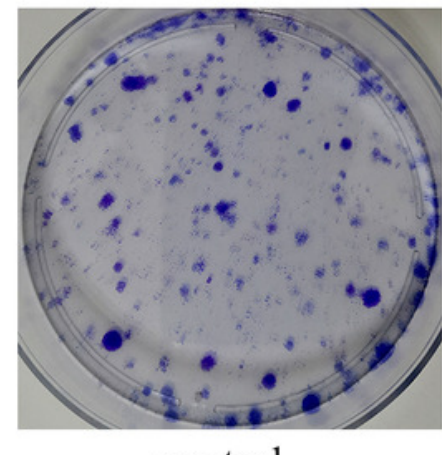

control

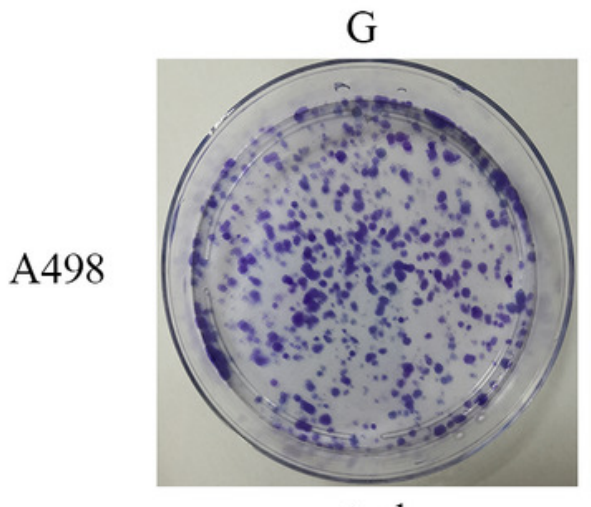

control
B

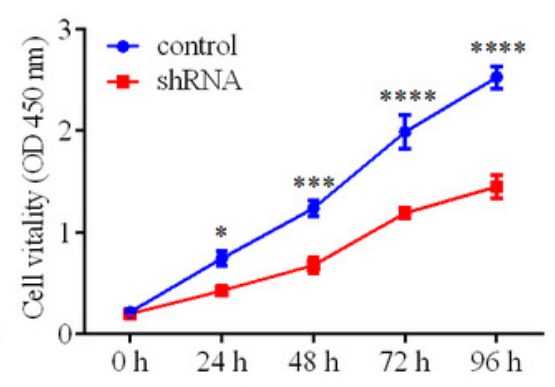

$\mathrm{C}$

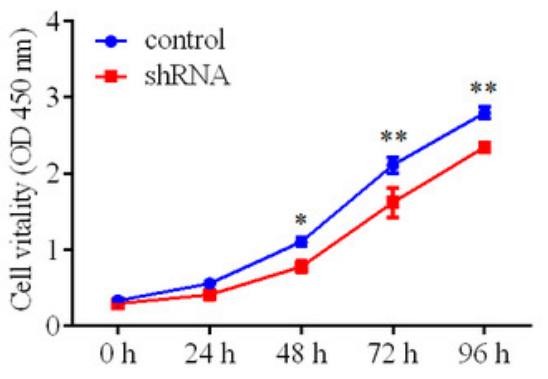

E

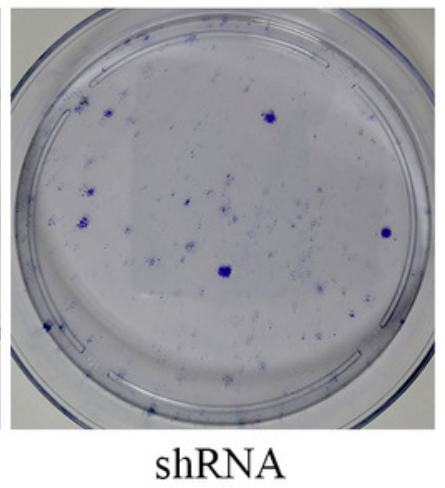

$\mathrm{H}$

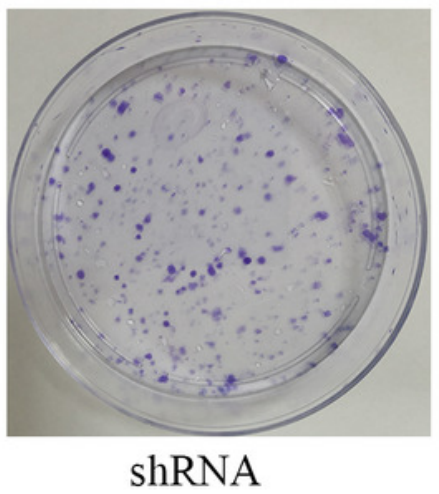

F
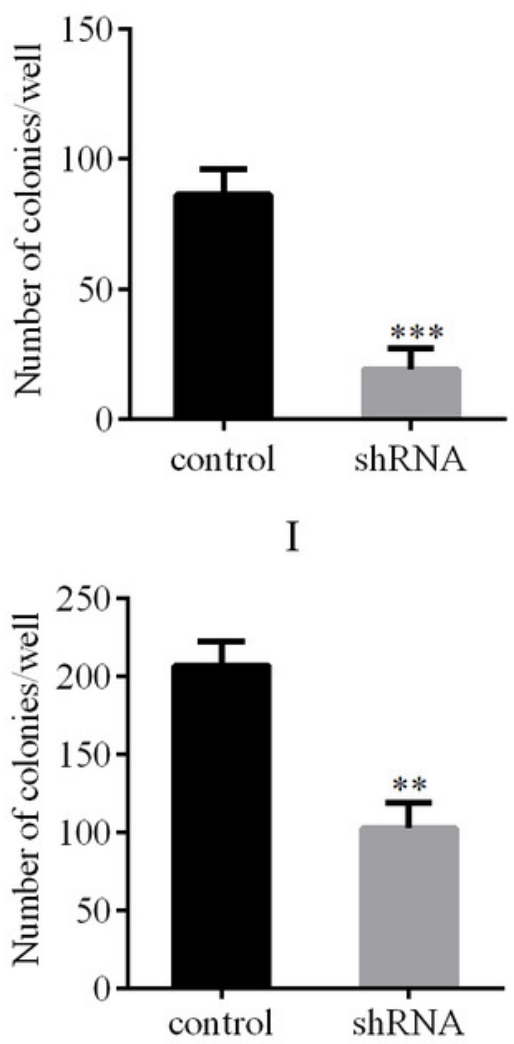


\section{Figure 7}

LINC01234 knockdown inhibited the migration and invasion of cCRCC cells.

Cell migration and invasion capacities were measured by Transwell assay. Representative images (magnification, $\times 100$ ) were presented. It indicated that the migration abilities of Caki-2 ( A-C ) and A498 ( D-F) cells were reduced following LINC01234 knockdown (all $p<$ 0.05). The invasion abilities of Caki-2 ( $\mathrm{G}-\mathrm{I}$ ) and A498 ( $\mathrm{J}-\mathrm{L}$ ) cells were also inhibited following LINC01234 knockdown (all $p<0.05$ ). ${ }^{* * * *}, p<0.0001 ; c C R C C$, clear cell renal cell carcinoma. 


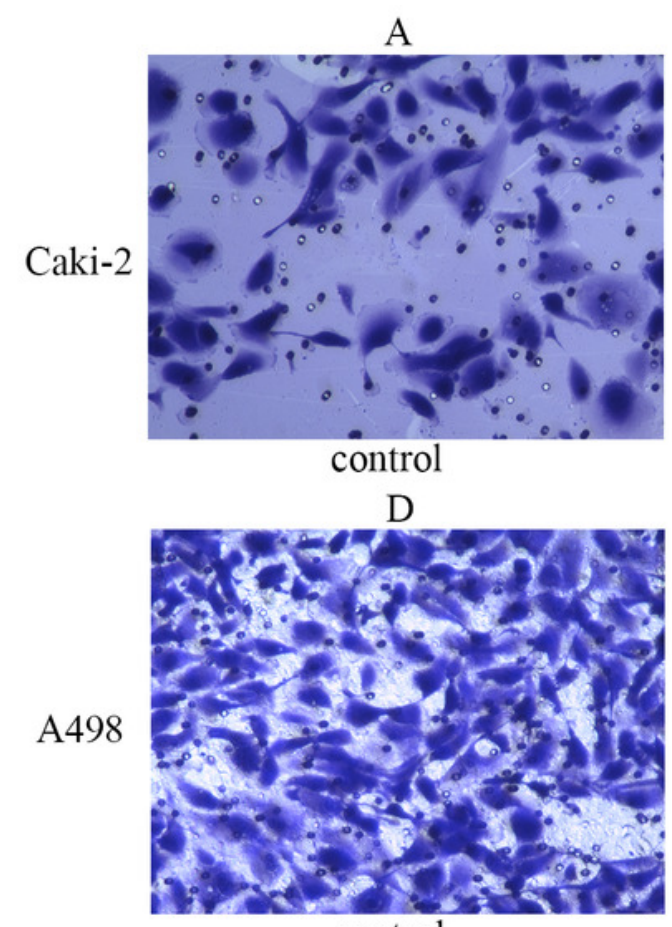

control

G

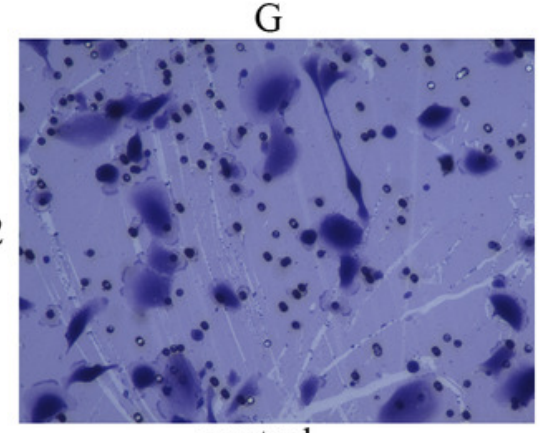

control

Caki-2

A498

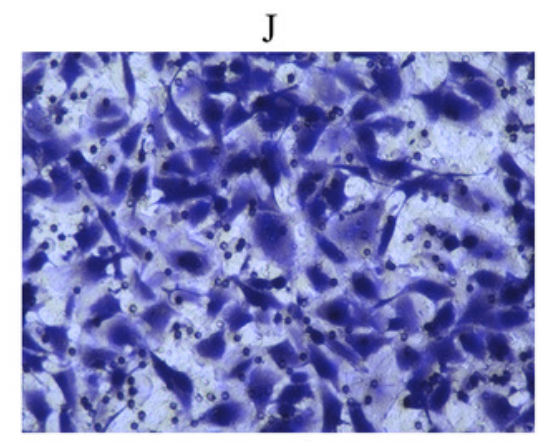

control
B

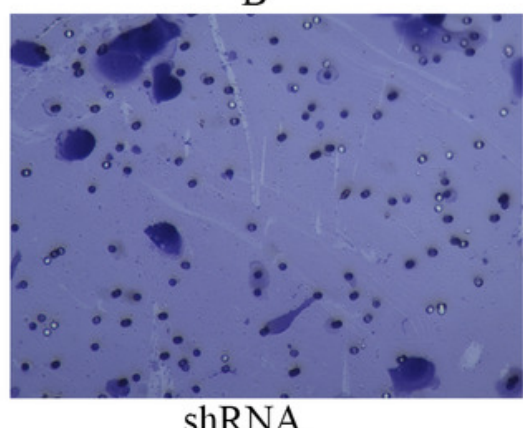

$\mathrm{E}$

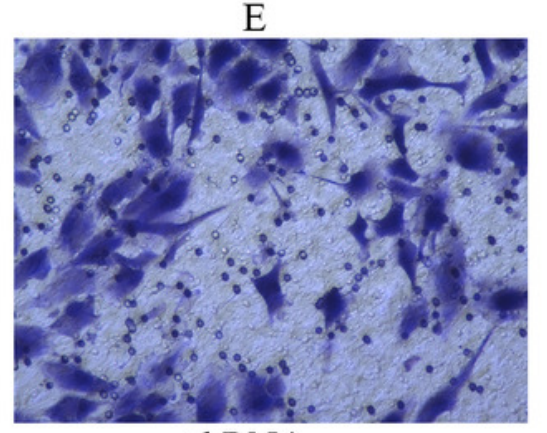

shRNA

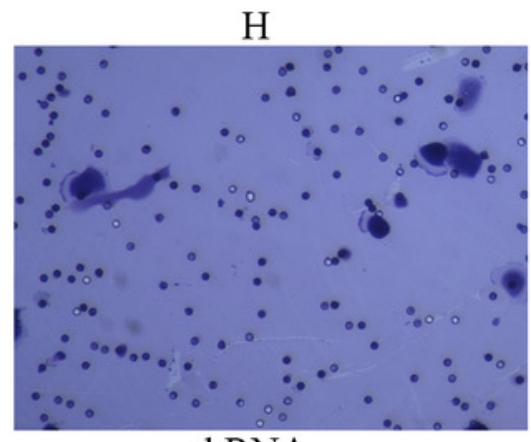

shRNA

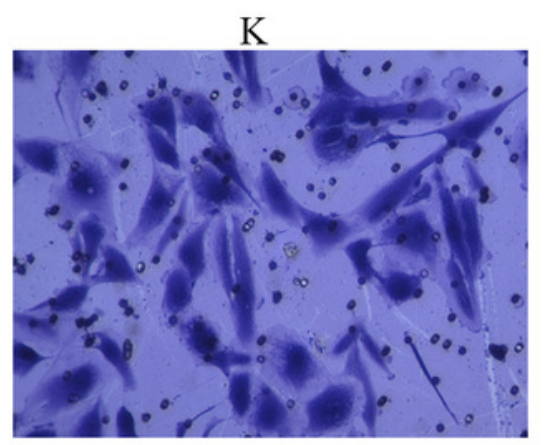

shRNA
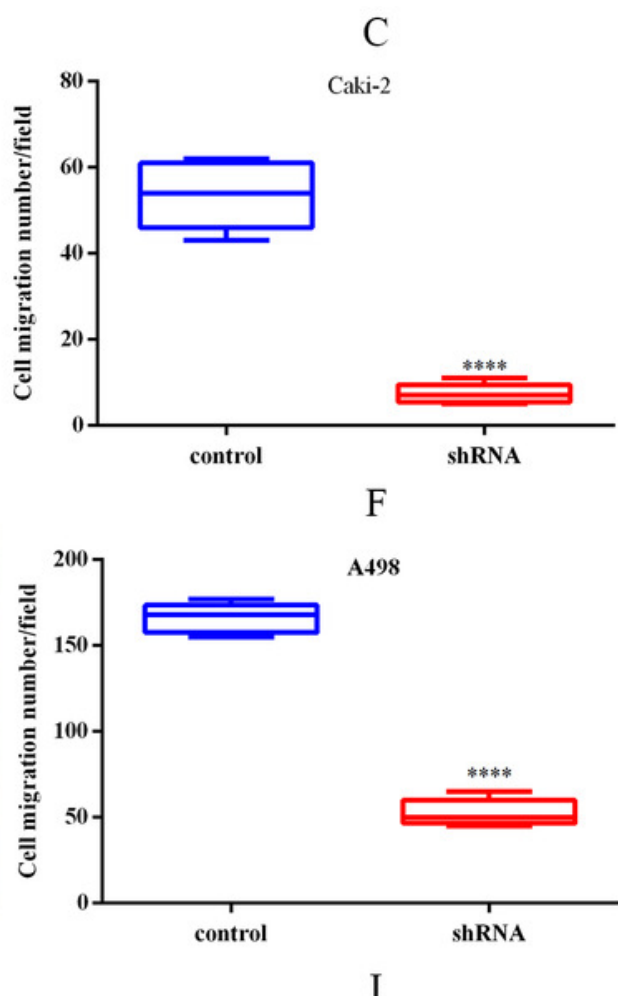

Caki-2

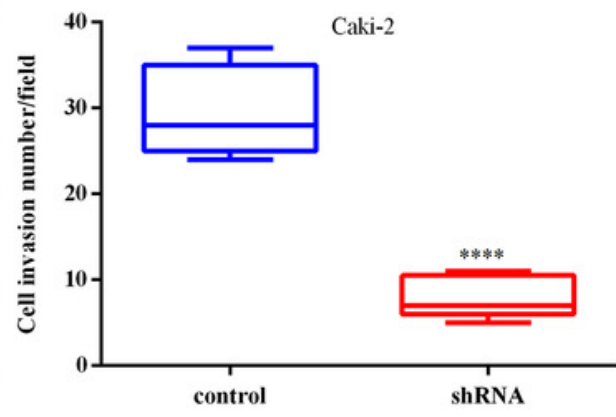

L

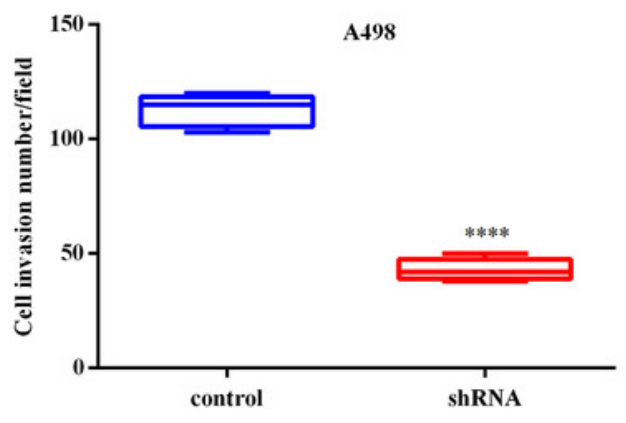




\section{Figure 8}

EMT process and HIF-2 $\alpha$ pathways were inhibited in cCRCC cells following LINC01234 knockdown.

$\square A, B \square$ The mRNA levels of N-cadherin, HIF-2 $\alpha$ and VEGFA were reduced, while that of Ecadherin was upregulated in cCRCC cells with LINC01234 knockdown. $\square C \square$ EMT process was inhibited in cCRCC cells with LINC01234 knockdown. The protein expression levels of $\beta$ catenin, Snail, N-cadherin and Vimentin were reduced, while that of E-cadherin was increased in Caki-2 and A498 cells with LINC01234 knockdown. पDQ HIF-2a pathway was inhibited in cCRCC cells with LINC01234 knockdown. The protein expression levels of HIF-1 $\alpha, H I F-2 \alpha$, VEGFA, EGFR, C-Myc, Cyclin D1 and MET were reduced in A498 and Caki-2 cells with LINC01234 knockdown. EMT, epithelial-mesenchymal transition; cCRCC, clear cell renal cell carcinoma. 
A

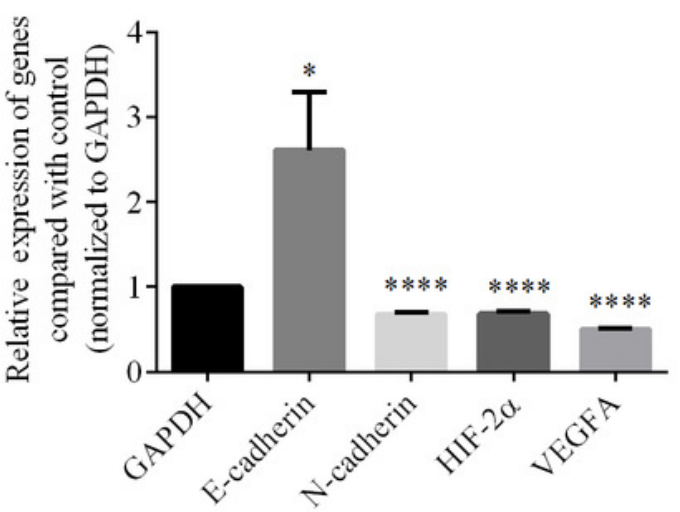

C

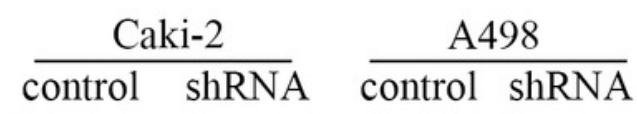

\section{$\beta$-actin}

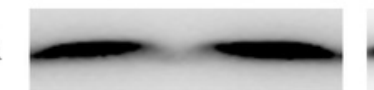

$\beta$-catenin

Vimentin
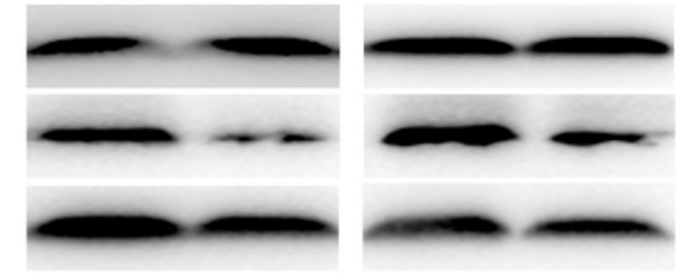

Vimentin

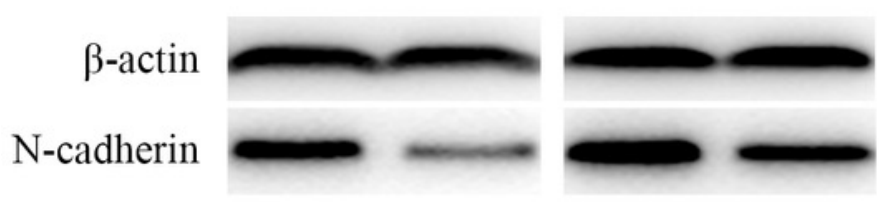

$\beta$-actin
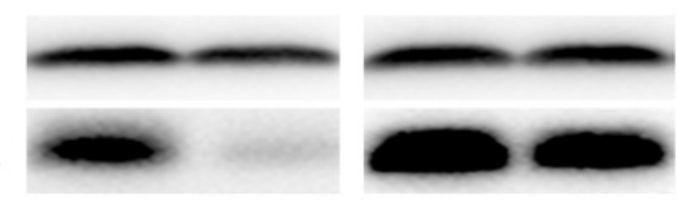

Snail

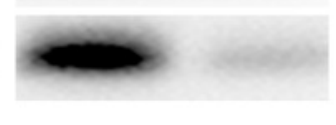

\section{GAPDH}

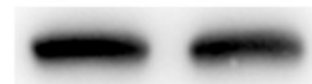

E-cadherin
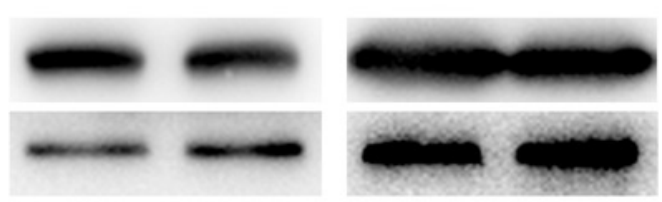

B

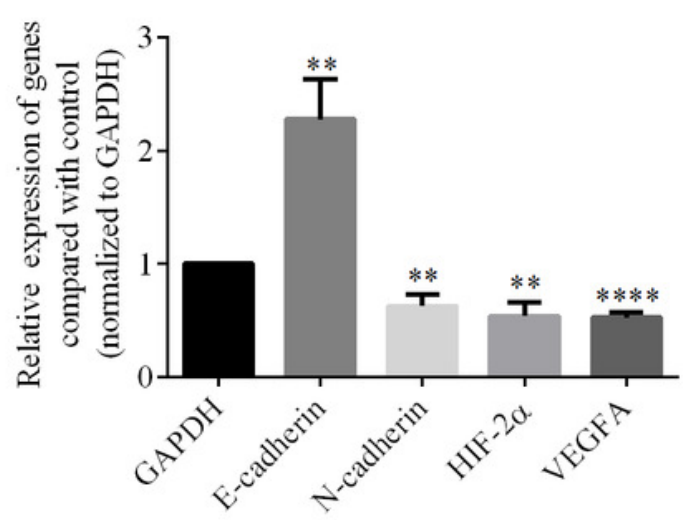

$\mathrm{D}$

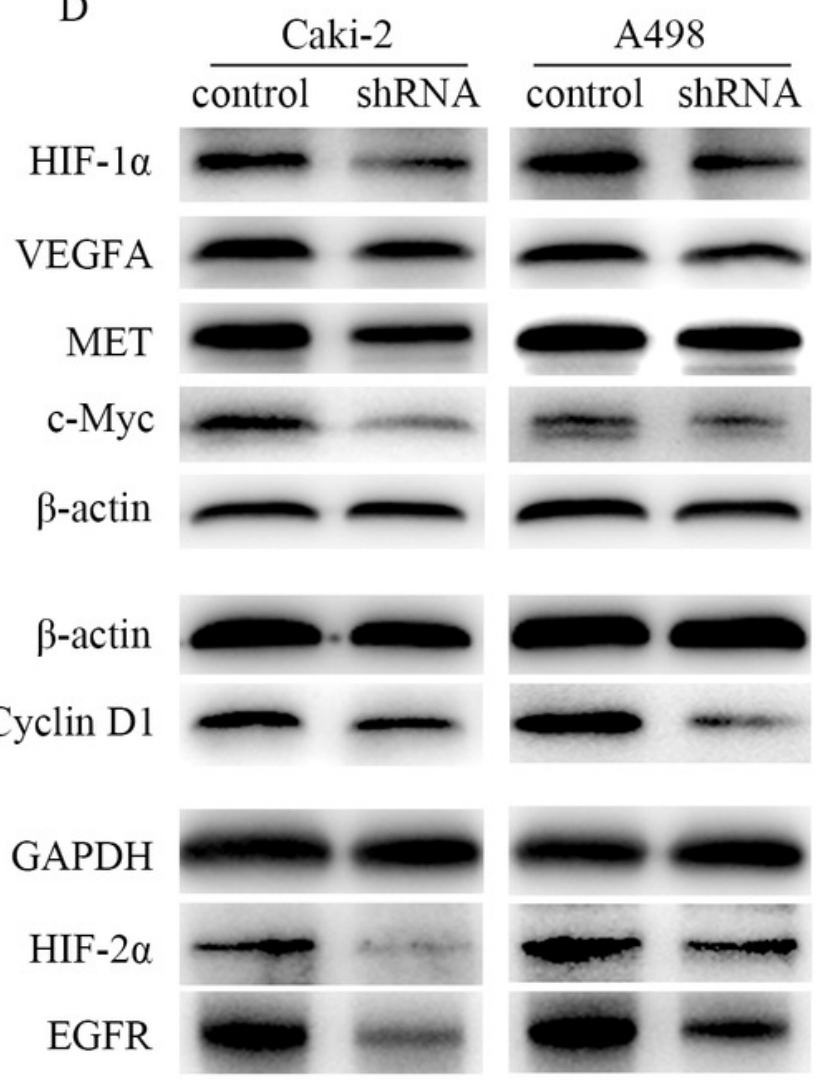

